\title{
HOMOLOGICAL THEORY OF IDEMPOTENT IDEALS
}

\author{
M. AUSLANDER, M. I. PLATZECK AND G. TODOROV
}

\begin{abstract}
Let $\Lambda$ be an artin algebra $\mathfrak{A}$ and a two-sided ideal of $\Lambda$. Then $\mathfrak{A}$ is the trace of a projective $\Lambda$-module $P$ in $\Lambda$. We study how the homological properties of the categories of finitely generated modules over the three rings $\Lambda / \mathfrak{A}, \Lambda$ and the endomorphism ring of $P$ are related. We give some applications of the ideas developed in the paper to the study of quasi-hereditary algebras.
\end{abstract}

\section{INTRODUCTION}

Throughout this paper we assume that $\Lambda$ is an artin algebra and all $\Lambda$ modules are assumed to lie in $\bmod \Lambda$, the category of finitely generated left $\Lambda$-modules.

In connection with their definition of quasi-hereditary algebras, Cline, Parshall and Scott [CPS] introduced the notion of an heredity ideal which is defined as follows. An heredity ideal of $\Lambda$ is a two-sided ideal $\mathfrak{A}$ satisfying the following conditions: (i) $\mathfrak{A}$ is idempotent (i.e. $\mathfrak{A}^{2}=\mathfrak{A}$ ), (ii) $\mathfrak{A}$ is projective as a left $\Lambda$-module and (iii) $\operatorname{End}_{\Lambda}(\mathfrak{A})$ is semisimple. The results concerning heredity ideals proven in [CPS, DR 1,2, BF] suggested studying the homological properties of idempotent ideals in a broader context. This paper is a preliminary step in this direction.

Our starting point is the following easily checked well-known observation. Let $P$ be a projective $\Lambda$-module. Then $\mathfrak{A}=\tau_{P}(\Lambda)$, the trace of $P$ in $\Lambda$ which is the ideal generated by the homomorphic images of $P$ in $\Lambda$, is an idempotent ideal in $\Lambda$ and one obtains all the idempotent ideals of $\Lambda$ this way. Moreover if $P$ and $Q$ are projective $\Lambda$-modules, then $\tau_{P}(\Lambda)=\tau_{Q}(\Lambda)$ if and only if add $P=\operatorname{add} Q$, where add $M$, for an arbitrary $\Lambda$-module $M$, is the full subcategory of $\bmod \Lambda$ consisting of all modules isomorphic to summands of finite sums (direct) of $M$. Therefore associated with a projective $\Lambda$-module $P$ is the idempotent ideal $\mathfrak{A}_{P}=\tau_{P}(\Lambda)$ and the artin algebras $\Lambda / \mathfrak{A}_{P}$ and $\Gamma_{P}=$ $\operatorname{End}(P)^{\mathrm{op}}$. Our basic aim is to study how the homological properties of the three categories $\bmod \Lambda / \mathfrak{A}_{P}, \bmod \Lambda$ and $\bmod \Gamma_{P}$ are related. It is worth noting, that

Received by the editors August 1, 1989 and, in revised form, May 11, 1990.

1980 Mathematics Subject Classification (1985 Revision). Primary 16A64; Secondary 16A35, $16 \mathrm{~A} 62$.

Key words and phrases. idempotent ideal, projective resolution, quasihereditary algebra.

The first author's work supported by NSF Grant No. DMS-8603487.

The second author's work supported by a fellowship of the Consejo Nacional de Investigaciones Científicas y Técnicas de la República Argentina. 
since $\Lambda / \mathfrak{A}_{P}$ and $\Gamma_{P}$ have fewer simple modules than $\Lambda$, a good understanding of the relationship between the categories $\bmod \Lambda / \mathfrak{A}_{P}, \bmod \Lambda$ and $\bmod \Gamma_{P}$ should lead to a way of studying $\bmod \Lambda$ in terms of algebras with fewer simple modules.

One relationship of interest is the inclusion functor $\bmod \Lambda / \mathfrak{A}_{P} \rightarrow \bmod \Lambda$. Saying that $\mathfrak{A}_{P}$ is an idempotent ideal is equivalent to saying that $\bmod \Lambda / \mathfrak{A}_{P}$ is a Serre subcategory of $\bmod \Lambda$. Moreover the exact functor $(P):, \bmod \Lambda \rightarrow$ $\bmod \Gamma_{P}$ given by $M \mapsto \operatorname{Hom}_{\Lambda}(P, M)$ gives an equivalence of categories between $\bmod \Gamma_{P}$ and the quotient of $\bmod \Lambda$ by $\bmod \Lambda / \mathfrak{A}_{P}$. In other words, we have an exact sequence of categories

$$
\bmod \Lambda / \mathfrak{A}_{P} \rightarrow \bmod \Lambda \rightarrow \bmod \Gamma_{P} .
$$

It is this exact sequence which is our main object of study.

We first concentrate on the inclusion $\bmod \Lambda / \mathfrak{A}_{P} \rightarrow \bmod \Lambda$. For any twosided ideal $\mathfrak{A}$ in $\Lambda$, we have a morphism of connected sequences of functors $\operatorname{Ext}_{\Lambda / \mathfrak{A}}^{i}(X, Y) \rightarrow \operatorname{Ext}_{\Lambda}^{i}(X, Y)$ for all $X$ and $Y$ in $\bmod \Lambda / \mathfrak{A}$ induced by the canonical isomorphism of functors $\operatorname{Hom}_{\Lambda / \mathfrak{A}}(X, Y) \stackrel{\sim}{\rightarrow} \operatorname{Hom}_{\Lambda}(X, Y)$ for all $X$ and $Y$ in $\bmod \Lambda / \mathfrak{A}$. It is well known that $\operatorname{Ext}_{\Lambda / \mathfrak{A}}^{1}(X, Y) \rightarrow \operatorname{Ext}_{\Lambda}^{1}(X, Y)$ is an isomorphism if and only if $\mathfrak{A}$ is an idempotent ideal. This suggests the following definitions. A two-sided ideal $\mathfrak{A}$ is a $k$-idempotent for $k \geq 1$ if $\operatorname{Ext}_{\Lambda / \mathfrak{A}}^{i}(X, Y) \rightarrow \operatorname{Ext}_{\Lambda}^{i}(X, Y)$ is an isomorphism for all $0 \leq i \leq k$ and all $X$ and $Y$ in $\bmod \Lambda / \mathfrak{A}$. We say that $\mathfrak{A}$ is a strong idempotent if $\operatorname{Ext}_{\Lambda}^{i}(X, Y) \rightarrow$ $\operatorname{Ext}_{\Lambda / \mathfrak{A}}^{i}(X, Y)$ is an isomorphism for all $i \geq 0$ and all $X$ and $Y$ in $\bmod \Lambda / \mathfrak{A}$. It is worth noting that if $\mathfrak{A}$ is an heredity ideal, then $\mathfrak{A}$ is a strong idempotent [DR 1, Part 2]. We give characterizations of $k$-idempotents in terms of projective and injective $\Lambda$-resolutions of $\Lambda / \mathfrak{A}$-modules.

Next we turn our attention to the functor $(P):, \bmod \Lambda \rightarrow \bmod \Gamma_{p}$. Since $(P, \quad)$ is exact we obtain a morphism of connected sequences of functors $\operatorname{Ext}_{\Lambda}^{i}(X, Y) \rightarrow \operatorname{Ext}_{\Gamma_{P}}^{i}((P, X),(P, Y))$ for all $X$ and $Y$ in $\bmod \Lambda$. In connection with studying when this is an isomorphism we introduce the following subcategories of $\bmod \Lambda$. For $k \geq 0$, we define $\mathbf{I}_{k+1}$ to be the full subcategory of $\bmod \Lambda$ consisting of all $Y$ in $\bmod \Lambda$ such that $\operatorname{Ext}_{\Lambda}^{i}(X, Y) \rightarrow$ $\operatorname{Ext}_{\Gamma_{P}}^{i}((P, X),(P, Y))$ is an isomorphism for all $0 \leq i \leq k$ and all $X$ in $\bmod \Lambda$. And we define $I_{\infty}$ to be the full subcategory of $\bmod \Lambda$ consisting of all $Y$ in $\bmod \Lambda$ such that $\operatorname{Ext}_{\Lambda}^{i}(X, Y) \rightarrow \operatorname{Ext}_{\Gamma_{P}}^{i}((P, X),(P, Y))$ is an isomorphism for all $i \geq 0$ and all $X$ in $\bmod \Lambda$. We characterize the subcategories $\mathbf{I}_{k}$ and $\mathbf{I}_{\infty}$ in terms of injective coresolutions of $\Gamma$-modules. In particular we consider the problem of when $\mathbf{I}_{k}=\mathbf{I}_{\infty}$. In this connection we show that an idempotent ideal $\mathfrak{A}$ is a projective $\Lambda$-module if and only if $\mathfrak{A}$ is a strong idempotent and $\mathbf{I}_{1}=\mathbf{I}_{\infty}$.

As an illustration of how these ideas and results can be used to deduce information about $\Lambda$ from the algebras $\Lambda / \mathfrak{A}$ and $\Gamma_{P}$ we prove the following generalization of a similar theorem about quasi-hereditary algebras proved in [CPS and DR1].

Let $\mathfrak{A}$ be an idempotent ideal. Suppose that $\operatorname{pd} \mathfrak{A}=r, \operatorname{gl} \operatorname{dim} \Lambda / \mathfrak{A}=s$ and gl. $\operatorname{dim} \Gamma=t$. Then $\mathrm{gl} \cdot \operatorname{dim} \Lambda \leq r+s+t+2$.

The paper ends with applications of these ideas to the study of quasihereditary algebras. In addition to giving proofs of some known results, we give a new description of quasihereditary algebras in terms of $l$-hereditary algebras. 


\section{Strong AND $k$-IDEMPOTENT IDEALS}

In this section we study the inclusion functor $\bmod \Lambda / \mathfrak{A} \rightarrow \bmod \Lambda$, where $\mathfrak{A}$ is an idempotent ideal in $\Lambda$. By an idempotent ideal we mean a two-sided ideal which is idempotent.

Let $\mathfrak{A}$ be a two-sided ideal of $\Lambda$. There is a map between connected sequences of functors $\varphi=\left(\varphi^{i}\right)_{i \geq 0}$ with $\varphi_{X, Y}^{i}: \operatorname{Ext}_{\Lambda / \mathfrak{A}}^{i}(X, Y) \rightarrow \operatorname{Ext}_{\Lambda}^{i}(X, Y)$ for $i \geq 0$ and $X, Y$ in $\bmod \Lambda / \mathfrak{A}$, induced by the canonical isomorphism $\varphi_{X, Y}^{0}: \operatorname{Hom}_{\Lambda / \mathfrak{A}}(X, Y) \rightarrow \operatorname{Hom}_{\Lambda}(X, Y)$. Notice that the ideal $\mathfrak{A}$ is idempotent precisely when the map $\varphi_{X, Y}^{1}: \operatorname{Ext}_{\Lambda / \mathfrak{A}}^{1}(X, Y) \rightarrow \operatorname{Ext}_{\Lambda}^{1}(X, Y)$ is an isomorphism for all $X, Y$ in $\bmod \Lambda / \mathfrak{A}$. We say that a two-sided ideal $\mathfrak{A}$ is $k$-idempotent if the maps $\varphi_{X, Y}^{i}$ are isomorphisms for all $i=0,1, \ldots, k$, and all $X, Y$ in $\bmod \Lambda / \mathfrak{A}$. We say that $\mathfrak{A}$ is a strong idempotent ideal if the maps $\varphi_{X, Y}^{i}$ are isomorphisms for all $i \geq 0$ and all $X, Y$ in $\bmod \Lambda / \mathfrak{A}$. In this section we give several characterizations of strong and $k$-idempotent ideals which are central to the rest of the paper.

We observe that being a $k$-idempotent is a symmetric condition. That is ${ }_{\Lambda} \mathfrak{A}$ is a $k$-idempotent ideal if and only if $\mathfrak{A}_{\Lambda}$ is a $k$-idempotent ideal. This follows by duality, since $\operatorname{Ext}_{\Lambda \text { op }}^{i}(D X, D Y) \simeq \operatorname{Ext}_{\Lambda}^{i}(Y, X)$ for $X, Y$ in $\bmod \Lambda$, where $D: \bmod \Lambda \rightarrow \bmod \Lambda^{\mathrm{op}}$ denotes the usual duality for artin algebras.

As another consequence of the definition we have that $\operatorname{gl} \cdot \operatorname{dim} \Lambda / \mathfrak{A} \leq \operatorname{gl} . \operatorname{dim} \Lambda$ when $\mathfrak{A}$ is a strong idempotent.

Now we study the behavior of injective coresolutions in $\bmod \Lambda$, when we apply the right adjoint $\operatorname{Hom}_{\Lambda}(\Lambda / \mathfrak{A}):, \bmod \Lambda \rightarrow \bmod \Lambda / \mathfrak{A}$ of the inclusion $\bmod \Lambda / \mathfrak{A} \rightarrow \bmod \Lambda$.

We start by recalling some facts about traces of modules. Let $M, N$ be $\Lambda$ modules. We denote by $\tau_{M}(N)$ the trace of $M$ in $N$. That is, $\tau_{M}(N)$ is the submodule of $N$ generated by the images of morphisms from $M$ to $N$. Let $\mathfrak{A}$ be a two-sided ideal of $\Lambda$. Then there is a natural isomorphism of $\Lambda / \mathfrak{A}$-modules Q: $\operatorname{Hom}_{\Lambda}(\Lambda / \mathfrak{A}, X) \stackrel{\sim}{\rightarrow} \tau_{\Lambda / \mathfrak{A}}(X)$ given by $\boldsymbol{\Theta}(f)=f(\overline{1})$. If $I$ is an injective $\Lambda$ modfule, then $\tau_{\Lambda / \mathfrak{A}}(I) \simeq \operatorname{Hom}(\Lambda / \mathfrak{A}, I)$ is an injective $\Lambda / \mathfrak{A}$-module. Moreover, if $I$ is an injective envelope of a $\Lambda$-module $X$, then $\tau_{\Lambda / \mathfrak{A}}(I)$ is an injective envelope of the $\Lambda / \mathfrak{A}$-module $\tau_{\Lambda / \mathfrak{A}}(X)$.

We describe when the above property about injective envelopes can be extended to a statement about the first $k$ terms, or about all of the terms, of a minimal injective coresolution of $\tau_{\Lambda / \mathfrak{A}}(X)$ in $\bmod \Lambda / \mathfrak{A}$.

In order to avoid writing separate statements we will sometimes write $j \leq$ $i \leq k$ where $k$ is either a positive integer or $\infty$. By $j \leq i \leq \infty$ we mean all $i \geq j$.

Proposition 1.1. Let $X$ be a $\Lambda$-module, let $0 \rightarrow X \rightarrow I_{0} \rightarrow I_{1} \rightarrow \cdots$ be a minimal injective coresolution of $X$ and $1 \leq k \leq \infty$. Then the following are equivalent

(i) $0 \rightarrow \tau_{\Lambda / \mathfrak{A}} X \rightarrow \tau_{\Lambda / \mathfrak{A}} I_{0} \rightarrow \cdots \rightarrow \tau_{\Lambda / \mathfrak{A}} I_{k}$ is the beginning of a minimal injective coresolution of $\tau_{\Lambda / \mathfrak{A}}(X)$ in $\bmod \Lambda / \mathfrak{A}$.

(ii) $\operatorname{Ext}_{\Lambda}^{i}(\Lambda / \mathfrak{A}, X)=0$ for all $i$ such that $1 \leq i \leq k$.

(iii) Let $Y$ be in $\bmod \Lambda / \mathfrak{A}$ and let $\varphi_{X, Y}^{i}: \operatorname{Ext}_{\Lambda / \mathfrak{A}}^{i}\left(Y, \tau_{\Lambda / \mathfrak{A}} X\right) \rightarrow \operatorname{Ext}_{\Lambda}^{i}(Y, X)$ be the map of connected sequences of functors induced by the canonical isomor- 
phism $\operatorname{Hom}_{\Lambda / \mathfrak{A}}\left(Y, \tau_{\Lambda / \mathfrak{A}} X\right) \simeq \operatorname{Hom}_{\Lambda}(Y, X)$. Then $\varphi_{\Lambda / \mathfrak{A}}^{i}$ is an isomorphism for $1 \leq i \leq k$.

Proof. We have that $\operatorname{Ext}_{\Lambda}^{i}(\Lambda / \mathfrak{A}, X)=0$ for all $1 \leq i \leq k$ if and only if the complex obtained after applying $\operatorname{Hom}_{\Lambda}(\Lambda / \mathfrak{A}$,$) to 0 \rightarrow X \rightarrow I_{0} \rightarrow I_{1} \rightarrow \cdots \rightarrow$ $I_{k}$ is exact. The equivalence of (i) and (ii) follows, since $\operatorname{Hom}_{\Lambda}(\Lambda / \mathfrak{A}, \quad)$ and $\tau_{\Lambda / \mathfrak{A}}(\mathrm{)})$ are isomorphic functors.

For a $\Lambda / \mathfrak{A}$-module $Y$ the complex obtained by applying the functor $\operatorname{Hom}_{\Lambda}(Y$,$) to the complex 0 \rightarrow X \rightarrow I_{0} \rightarrow I_{1} \rightarrow \cdots$ is isomorphic to the complex obtained by applying $\operatorname{Hom}_{\Lambda / \mathfrak{A}}(Y, \quad)$ to $0 \rightarrow \tau_{\Lambda / \mathfrak{A}} X \rightarrow \tau_{\Lambda / \mathfrak{A}} I_{0} \rightarrow$ $\tau_{\Lambda / \mathfrak{A}} I_{1} \ldots$. So the homologies of the resulting complexes are isomorphic, proving that (i) $\Rightarrow$ (iii). Clearly (iii) $\Rightarrow$ (ii), so the proof is complete.

Dually, we can consider projective resolutions of $\Lambda$-modules and apply the left adjoint functor $\Lambda / \mathfrak{A} \otimes_{\Lambda}: \bmod \Lambda \rightarrow \bmod \Lambda / \mathfrak{A}$ to the inclusion $\bmod \Lambda / \mathfrak{A}$ $\rightarrow \bmod \Lambda$. We observe that $\Lambda / \mathfrak{A} \otimes_{\Lambda} X \simeq X / \mathfrak{A} X$. So we have the following proposition.

Proposition 1.2. Let $X$ be a $\Lambda$-module, let $\cdots \rightarrow P_{1} \rightarrow P_{0} \rightarrow X \rightarrow 0$ be a minimal projective resolution of $X$ and $1 \leq k \leq \infty$. Then the following are equivalent:

(i) $P_{k} / \mathfrak{A} P_{k} \rightarrow \cdots \rightarrow P_{0} / \mathfrak{A} P_{0} \rightarrow X / \mathfrak{A} X \rightarrow 0$ is the beginning of a minimal projective resolution of $X / \mathfrak{A} X$ in $\bmod (\Lambda / \mathfrak{A})$.

(ii) $\operatorname{Tor}_{i}^{\Lambda}(\Lambda / \mathfrak{A}, X)=0$ for all $i$ such that $1 \leq i \leq k$.

(iii) Let $Y$ in $\bmod (\Lambda / \mathfrak{A})^{\text {op }}$ and let $\psi_{i}^{X, Y}: \operatorname{Tor}_{i}^{\Lambda}(Y, X) \rightarrow \operatorname{Tor}_{i}^{\Lambda / \mathfrak{A}}(Y, X / \mathfrak{A} X)$ be the map of connected sequences of functors induced by the isomorphism $Y \otimes_{\Lambda} X$ $\simeq Y \otimes_{\Lambda / \mathfrak{A}} X / \mathfrak{A} X$. Then $\psi_{i}^{X, Y}$ is an isomorphism for $i=1, \ldots, k$.

From the above results we have the following characterization of $k$-idempotent ideals in terms of Ext and Tor functors.

Proposition 1.3. Let $\mathfrak{A}$ be a two-sided ideal. Let $1 \leq k \leq \infty$. Then the following conditions are equivalent:

(i) The ideal $\mathfrak{A}$ is k-idempotent.

(ii) $\varphi_{X / Y}^{i}: \operatorname{Ext}_{\Lambda / \mathfrak{A}}^{i}(X, Y) \rightarrow \operatorname{Ext}_{\Lambda}^{i}(X, Y)$ is an isomorphism for all $X, Y$ in $\bmod \Lambda / \mathfrak{A}$ and all $0 \leq i \leq k$.

(iii) $\operatorname{Ext}_{\Lambda}^{i}(\Lambda / \mathfrak{A}, Y)=0$ for all $\Lambda / \mathfrak{A}$-modules $Y$ and all $1 \leq i \leq k$.

(iv) $\operatorname{Ext}_{\Lambda}^{i}(\Lambda / \mathfrak{A}, I)=0$ for all injective $I$ in $\bmod \Lambda / \mathfrak{A}$ and $1 \leq i \leq k$.

(ii') $\varphi_{X, Y}^{i}: \operatorname{Tor}_{i}^{\Lambda / \mathfrak{A}}(X, Y) \rightarrow \operatorname{Tor}_{i}^{\Lambda}(X, Y)$ is an isomorphism for all $X$ in $\bmod (\Lambda / \mathfrak{A})^{\text {op }}$ and $Y$ in $\bmod \Lambda / \mathfrak{A}$ and all $0 \leq i \leq k$.

(iii') $\operatorname{Tor}_{i}^{\Lambda}(\Lambda / \mathfrak{A}, Y)=0$ for all $Y$ in $\bmod \Lambda / \mathfrak{A}$ and all $1 \leq i \leq k$.

(iv') $\operatorname{Tor}_{i}^{\Lambda}(\Lambda / \mathfrak{A}, \Lambda / \mathfrak{A})=0$ for all $1 \leq i \leq k$.

Proof. (i) $\Leftrightarrow$ (ii) is just the definition of $k$-idempotent ideals. Obviously (ii) $\Rightarrow$ (iii) $\Rightarrow$ (iv). By Proposition 1.1 it follows that (iii) $\Rightarrow$ (ii). (iv) $\Rightarrow$ (iii) Let $Y$ be a $\Lambda / \mathfrak{A}$-module, $I$ its injective envelope in $\bmod \Lambda / \mathfrak{A}$ and $0 \rightarrow Y \rightarrow$ $I \rightarrow I / Y \rightarrow 0$ exact. Applying $\operatorname{Hom}_{\Lambda}(\Lambda / \mathfrak{A}, \quad)$ to this sequence we get a commutative diagram:

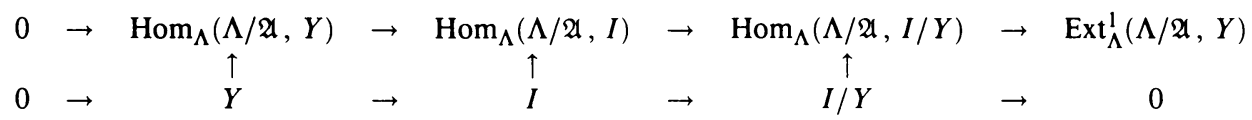


Since the vertical maps are isomorphisms we have a long exact sequence:

0

$$
\begin{aligned}
0 \longrightarrow \operatorname{Ext}_{\Lambda}^{1}(\Lambda / \mathfrak{A}, Y) & \longrightarrow \operatorname{Ext}_{\Lambda}^{1}(\Lambda / \mathfrak{A}, I) \longrightarrow \operatorname{Ext}_{\Lambda}^{1}(\Lambda / \mathfrak{A}, I / Y) \longrightarrow \operatorname{Ext}_{\Lambda}^{k}(\Lambda / \mathfrak{A}, Y) \longrightarrow{ }_{\Lambda}^{k-1}(\Lambda / \mathfrak{A}, I / Y) \longrightarrow \\
\cdots & \longrightarrow
\end{aligned}
$$

Then (iv) $\Rightarrow$ (iii) follows by induction on $k$.

For the other equivalences observe that $D\left(\operatorname{Ext}_{\Lambda}^{i}(X, Y)\right) \simeq \operatorname{Tor}_{i}^{\Lambda}(D Y, X)$, where $D: \bmod \Lambda \rightarrow \bmod \Lambda^{\text {op }}$ is the usual duality for artin algebras [CE, Chapter VI, §5].

We give a description of $k$-idempotent ideals where $k=1$ and 2 in terms of the multiplication map $\mathfrak{A} \otimes_{\Lambda} \mathfrak{A} \rightarrow \mathfrak{A}$. We do not have a similar description for higher $k$.

Lemma 1.4. Let $\mathfrak{A}$ be a two-sided ideal and let $m: \mathfrak{A} \otimes_{\Lambda} \mathfrak{A} \rightarrow \mathfrak{A}$ be the multiplication map. Then

(a) $\mathfrak{A}$ is idempotent if and only if $m$ is an epimorphism,

(b) $\mathfrak{A}$ is 2-idempotent if and only if $m$ is an isomorphism.

Proof. (a) is clear, and (b) follows from the following result from [CE, Exercise 19, p. 126], which we state for ease of reference.

Lemma 1.5. Let $\mathfrak{A}$ be an idempotent ideal. Then there is an exact sequence $0 \rightarrow \operatorname{Tor}_{2}^{\Lambda}(\Lambda / \mathfrak{A}, \Lambda / \mathfrak{A}) \rightarrow \mathfrak{A} \otimes_{\Lambda} \mathfrak{A} \stackrel{m}{\rightarrow} A \rightarrow 0$, where $m$ is the multiplication map.

We get the following characterization of $k$-idempotent ideals in terms of the projective and injective resolutions of $\Lambda / \mathfrak{A}$-modules.

Theorem 1.6. The following conditions are equivalent for a two-sided ideal $\mathfrak{A}$ :

(i) The ideal $\mathfrak{A}$ is k-idempotent.

(ii) Let $X$ be a $\Lambda / \mathfrak{A}$-module and $0 \rightarrow X \rightarrow I_{0} \rightarrow \cdots$ a minimal injective coresolution of $X$ in $\bmod \Lambda$. Then $0 \rightarrow X \rightarrow \tau_{\Lambda / \mathfrak{A}} I_{0} \rightarrow \cdots \rightarrow \tau_{\Lambda / \mathfrak{A}} I_{k}$ is the beginning of a minimal injective coresolution of $X$ in $\bmod \Lambda / \mathfrak{A}$.

(iii) Let $Y$ be a $\Lambda / \mathfrak{A}$-module and $\cdots \rightarrow P_{0} \rightarrow Y \rightarrow 0$ a minimal projective resolution of $Y$ in $\bmod \Lambda$. Then $P_{k} / \mathfrak{A} P_{k} \rightarrow \cdots \rightarrow P_{0} / \mathfrak{A} P_{0} \rightarrow Y \rightarrow 0$ is the beginning of a minimal projective resolution of $Y$ in $\bmod \Lambda / \mathfrak{A}$.

Proof. It was proven in Proposition 1.3 that $\mathfrak{A}$ is $k$-idempotent is equivalent to $\operatorname{Ext}_{\Lambda}^{i}(\Lambda / \mathfrak{A}, Y)=0$ for all $\Lambda / \mathfrak{A}$-modules $Y$ and $1 \leq i \leq k$. The condition (ii) in the theorem is part of the statement of Proposition 1.1 for $Y$ in $\bmod \Lambda / \mathfrak{A}$. Similarly $\mathfrak{A}$ being $k$-idempotent is equivalent to $\operatorname{Tor}_{i}^{\Lambda}(\Lambda / \mathfrak{A}, Y)=0$ for all $Y$ in $\bmod \Lambda / \mathfrak{A}$ and $1 \leq i \leq k$ which is equivalent to (iii) by Proposition 1.2.

Next we give several examples of strong and $k$-idempotent ideals.

Example 1. Let $\mathfrak{A}$ be an idempotent ideal which is a projective left $\Lambda$-module. Then $\mathfrak{A}$ is a strong idempotent ideal. To see this, let $Y$ be a $\Lambda / \mathfrak{A}$-module. Then $\operatorname{Ext}_{\Lambda}^{i}(\Lambda / \mathfrak{A}, Y)=0$ for all $i \geq 1$. This is true for $i=1$ because $\mathfrak{A}$ is an idempotent ideal, and for $i>1$ because the projective dimension of $\Lambda / \mathfrak{A}$ is at most 1 .

Therefore, if $\Lambda$ is a hereditary algebra any idempotent ideal is strong idempotent. It would be nice to know what other algebras $\Lambda$ contain nontrivial idempotent ideals which are projective left $\Lambda$-modules. Nonsimple quasi-hereditary algebras do, since heredity ideals are projective left modules, by definition. (See [CPS, DR 1] or $\S 6$ for definitions.) 
Example 2. The ideals which occur in a heredity chain in a quasihereditary algebra are all strong idempotent. More generally, let $\Lambda$ be an artin algebra having a chain of idempotent ideals $0=\mathfrak{A}_{0} \subset \mathfrak{A}_{1} \subset \cdots \subset \mathfrak{A}_{n} \subset \Lambda$ such that $\mathfrak{A}_{i} / \mathfrak{A}_{i-1}$ is projective $\Lambda / \mathfrak{A}_{i-1}$-module for all $i=1, \ldots, n$. Then $\mathfrak{A}_{i}$ is strong idempotent for $i=1, \ldots, n$.

This can be proven in a straightforward way by induction on $n$. Since it will also follow from results in $\S 6$ we omit the proof here.

In the above examples the projective dimension of the strong idempotent ideals was always finite. We give now an example of a strong idempotent ideal of infinite projective dimension.

Example 3. Let $\Lambda$ be the path algebra (over a field) of the quiver

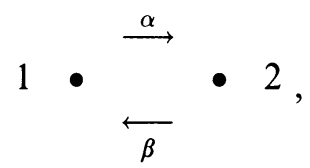

modulo the ideal generated by the relation $\beta \alpha \beta=0$. Let $P$ be the projective corresponding to the vertex 1 . Then $\mathfrak{A}=\tau_{P}(\Lambda)$ is a strong idempotent ideal and has infinite projective dimension.

The following example shows that for every natural number $k \geq 1$, there are $k$-idempotent ideals which are not $(k+1)$-idempotent.

Example 4. Let $\Lambda$ be the path algebra (over a field) of the quiver

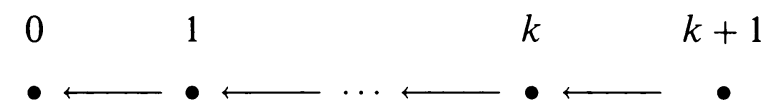

modulo the ideal generated by the paths of length 2 . Let $P_{i}$ be the projective corresponding to the vertex $i$. Let $\mathfrak{A}=\tau_{P_{2} \amalg \cdots \amalg P_{k}}(\Lambda)$. Then $\mathfrak{A}$ is $k$-idempotent and is not $(k+1)$-idempotent.

To see this we denote the simple $\Lambda$-modules by $S_{j}=P_{j} / \underline{r} P_{j}$, where $\underline{r}$ is the radical of $\Lambda$. Then $\Lambda / \mathfrak{A} \simeq S_{0} \amalg S_{k+1}$ is semisimple. So the only indecomposable $\Lambda / \mathfrak{A}$-module which is not an injective $\Lambda$-module is $S_{0}$. We have that $\operatorname{Ext}_{\Lambda}^{i}\left(\Lambda / \mathfrak{A}, S_{0}\right)=\operatorname{Ext}_{\Lambda}^{i}\left(S_{k+1}, S_{0}\right)$ for $i \geq 1$. But $\operatorname{Ext}_{\Lambda}^{i}\left(S_{k+1}, S_{0}\right)=0$ for $i \leq k$ and $\operatorname{Ext}_{\Lambda}^{k+1}\left(S_{k+1}, S_{0}\right) \neq 0$. It follows that $\mathfrak{A}$ is $k$-idempotent but is not $(k+1)$-idempotent.

Notice that the above algebra $\Lambda$ can be given as the $(k+2) \times(k+2)$ lower triangular matrix ring modulo the square of the radical.

\section{Projective Resolutions of $k$-iDempotent ideals}

In the previous section we characterized $k$-idempotent ideals in terms of the projective resolutions of all $\Lambda / \mathfrak{A}$-modules. We show here that knowing the projective resolution of only one module, namely ${ }_{\Lambda} \mathfrak{A}$, is enough to determine for which $k$ the ideal $\mathfrak{A}$ is $k$-idempotent. In this section we prove the following theorem.

Theorem 2.1. Let $\mathfrak{A}=\tau_{P}(\Lambda)$ for some projective $\Lambda$-module $P$ and $1 \leq k \leq$ $\infty$. Let $\cdots \rightarrow P_{i} \rightarrow \cdots \rightarrow P_{0} \rightarrow \mathfrak{A} \rightarrow 0$ be a minimal projective resolution 
of $\mathfrak{A}$. Then $\mathfrak{A}$ is $(k+1)$-idempotent if and only if the $P_{i}$ are in add $P$ for $i=0, \ldots, k$.

In the proof of the theorem and throughout the rest of the paper the following facts and notation will be used. A two-sided ideal $\mathfrak{A}$ is idempotent if and only if $\mathfrak{A}=\tau_{P}(\Lambda)$ for some projective $\Lambda$-module $P$. Furthermore, $\tau_{P}(\Lambda)=\tau_{P^{\prime}}(\Lambda)$ if and only if add $P=\operatorname{add} P^{\prime}$. From now on, $\mathfrak{A}$ will always denote an idempotent ideal, and $P$ a fixed projective $\Lambda$-module such that $\mathfrak{A}=\tau_{P}(\Lambda)$. We now recall some properties of traces of projective modules.

Remark 2.2. Let $\mathfrak{A}=\tau_{P}(\Lambda)$. Then $\tau_{P}(X)=\tau_{P}(\Lambda) X=\mathfrak{A} X$ for any $\Lambda$-module $X$. The projective cover $P_{0}(X)$ of $X$ is in add $P$ if and only if $X=\tau_{P}(X)=$ $\mathfrak{A} X$. We also have add $P=\operatorname{add}\left(P_{0}(\mathfrak{A})\right)$.

In order to prove the theorem it will be convenient to introduce the following notation:

Definition 2.3. Let $P$ be a projective $\Lambda$-module. For each $0 \leq k \leq \infty$ we define $\mathbf{P}_{k}$ to be the full subcategory of $\bmod \Lambda$ consisting of the $\Lambda$-modules $X$ having a projective resolution $\cdots \rightarrow P_{1} \rightarrow P_{0} \rightarrow X \rightarrow 0$ with $P_{i}$ in add $P$ for $0 \leq i \leq k$. Since $\mathbf{P}_{k}$ depencis only on add $P$, it is well defined for the idempotent ideal $\mathfrak{A}=\tau_{P}(\Lambda)$. It would be more precise to write $\mathbf{P}_{k, P}$ or $\mathbf{P}_{k, \mathfrak{A}}$, but whenever it is clear which projective or which idempotent ideal we are using, we will use the above notation $\mathbf{P}_{k}$.

Theorem 2.1 can now be restated.

Theorem 2.1'. An idempotent ideal $\mathfrak{A}=\tau_{P}(\Lambda)$ is $(k+1)$-idempotent if and only if $\mathfrak{A}$ is in $\mathbf{P}_{k}$, for $1 \leq k \leq \infty$.

In the following proposition we give a characterization of the modules in $\mathbf{P}_{k}$ needed in the proof of the above theorem as well as in the rest of the paper.

Proposition 2.4. Let $\mathfrak{A}=\tau_{P}(\Lambda)$ and $1 \leq k \leq \infty$. Then the following conditions are equivalent for a $\Lambda$-module $X$.

(i) $X$ is in $\mathbf{P}_{k}$.

(ii) $\operatorname{Ext}_{\Lambda}^{i}(X, Y)=0$ for all $\Lambda / \mathfrak{A}$-modules $Y$ and $i=0, \ldots, k$.

(iii) $\operatorname{Ext}_{\Lambda}^{i}(X, E)=0$ for all injective $\Lambda / \mathfrak{A}$-modules $E$ and $i=0, \ldots, k$.

Proof. By induction on $k$.

Proof of Theorem 2.1' (and Theorem 2.1). Let $X=\mathfrak{A}$ in the above proposition. We only need to observe that $\operatorname{Ext}_{\Lambda}^{i+1}(\Lambda / \mathfrak{A}, Y) \simeq \operatorname{Ext}_{\Lambda}^{i}(\mathfrak{A}, Y)$ for all $i \geq 0$ and use that $\mathfrak{A}$ is $(k+1)$-idempotent if and only if $\operatorname{Ext}_{\Lambda}^{i+1}(\Lambda / \mathfrak{A}, Y)=0$ for all $Y$ in $\bmod \Lambda / \mathfrak{A}$ and $i=0, \ldots, k$.

Dually, for $0 \leq k \leq \infty$ we define $\mathbf{I}_{k}$ to be the full subcategory of $\bmod \Lambda$ consisting of the $\Lambda$-modules $Y$ having an injective coresolution $0 \rightarrow Y \rightarrow$ $I_{0} \rightarrow I_{1} \rightarrow \cdots$ with $I_{i}$ in add $I$ for $0 \leq i \leq k$. Here $I$ denotes the injective envelope of $P / \underline{r} P$.

Using Theorem $2.1^{\prime}$ and the duality $D$, we see again that there is a module such that its injective resolution determines for which $k$ the ideal $\mathfrak{A}$ is $k$ idempotent.

Theorem 2.5. Let $M=E / E^{\prime}$, where $E$ is the sum of all nonisomorphic indecomposable injective $\Lambda$-modules and $E^{\prime}$ is the sum of all nonisomorphic 
indecomposable injective $\Lambda / \mathfrak{A}$-modules. Let $1 \leq k \leq \infty$. Then $M$ is in $\mathbf{I}_{k}$ if and only if $\mathfrak{A}$ is $(k+1)$-idempotent.

Notice that the module $M$ is the dual of $\mathfrak{A}$ considered as a right $\Lambda$-module.

We now write the dual statement of Proposition 2.4, since it will be used in the rest of the paper.

Proposition 2.6. Let $\mathfrak{A}=\tau_{P}(\Lambda)$ and $1 \leq k \leq \infty$. Then the following conditions are equivalent for a $\Lambda$-module $Y$ :

(i) $Y$ is in $\mathbf{I}_{k}$.

(ii) $\operatorname{Ext}_{\Lambda}^{i}(X, Y)=0$ for all $X$ in $\bmod \Lambda / \mathfrak{A}$ and all $i=0, \ldots, k$.

(iii) $\operatorname{Ext}_{\Lambda}^{i}(\Lambda / \mathfrak{A}, Y)=0$ for all $i=0, \ldots, k$.

\section{EXTENSIONS OVER THE ENDOMORPHISM RING OF $P$}

Let $\mathfrak{A}=\tau_{P}(\Lambda)$ be an idempotent ideal and $\Gamma=\operatorname{End}_{\Lambda}(P)$. It would be more precise to write $\Gamma_{P}$, but we have assumed in $\S 2$ that $P$ is a fixed projective module throughout the rest of the paper, so we just write $\Gamma$.

In $\S 1$ we considered the inclusion $\bmod \Lambda / \mathfrak{A} \rightarrow \bmod \Lambda$, and studied conditions on $\mathfrak{A}$ so that $\operatorname{Ext}_{\Lambda}^{i}(X, Y) \simeq \operatorname{Ext}_{\Lambda / \mathfrak{A}}^{i}(X, Y)$ for all $i=0,1, \ldots, k$. Now we turn our attention to the functor $(P):, \bmod \Lambda \rightarrow \bmod \Gamma$. This functor induces natural morphisms $\rho_{X, Y}^{i}: \operatorname{Ext}_{\Lambda}^{i}(X, Y) \rightarrow \operatorname{Ext}_{\Gamma}^{i}((P, X),(P, Y))$, for all $i \geq 0$ and all $X, Y$ in $\bmod \Lambda$. We will study in this section conditions under which these morphisms are isomorphims for all $i=0, \ldots, k$. And, also in analogy with what we did for $\Lambda / \mathfrak{A}$-modules and $\Lambda$-modules, we will explore the relationship between injective coresolutions in $\bmod \Gamma$ and in $\bmod \Lambda$.

We start by recalling some well-known properties of the functor $(P):, \bmod \Lambda$ $\rightarrow \bmod \Gamma$.

Let $\mathbf{I}_{k}$ and $\mathbf{P}_{k}$ be as in $\S 2$, for $0 \leq k \leq \infty$. Then $\mathbf{P}_{1}$ consists of the $\Lambda$-modules with a projective presentation in $\operatorname{add} P$, and $\mathbf{I}_{1}$ of those with an injective copresentation in add $I$. We will use the following results $[\mathrm{A}, \S \S 5$ and $6]$.

Lemma 3.1. (1) The functor $(P$,$) induces equivalences of categories$

$$
\left.(P,)\right|_{\mathbf{P}_{1}}: \mathbf{P}_{1} \rightarrow \bmod \Gamma,\left.\quad(P,)\right|_{\mathbf{I}_{1}}: \mathbf{I}_{1} \rightarrow \bmod \Gamma .
$$

(2) Let $\rho_{X, Y}:(X, Y) \rightarrow((P, X),(P, Y))$ be the map induced by $(P$,$) .$ Then

(i) $\rho_{X, Y}$ is a monomorphism if either $X$ is in $\mathbf{P}_{0}$ or $Y$ is in $\mathbf{I}_{0}$.

(ii) $\rho_{X, Y}$ is an isomorphism if $X$ is in $\mathbf{P}_{0}$ and $Y$ is $\mathbf{I}_{0}$.

(iii) $\rho_{X, Y}$ is an isomorphism if either $X$ is in $\mathbf{P}_{1}$ or $Y$ is in $\mathbf{I}_{1}$.

(3) $(P, \quad)$ induces equivalences of categories between add $P$ and the category of projective $\Gamma$ modules and between add $I$ and the category of injective $\Gamma$ modules.

Since $(P):, \bmod \Lambda \rightarrow \bmod \Gamma$ is an exact functor, it induces morphisms $\rho_{X, Y}^{i}: \operatorname{Ext}_{\Lambda}^{i}(X, Y) \rightarrow \operatorname{Ext}_{\Lambda}^{i}((P, X),(P, Y))$, for $X, Y$ in $\bmod \Lambda, i \geq 0$, functorial in $X$ and $Y$. These morphisms are defined as follows. Let $0 \rightarrow$ $Y \rightarrow I_{0} \rightarrow I_{1} \rightarrow \cdots$ be an injective copresentation of $Y$ in $\bmod \Lambda$. Then the exact complex $0 \rightarrow(P, Y) \rightarrow\left(P, I_{0}\right) \rightarrow\left(P, I_{1}\right) \rightarrow \cdots$ maps into an exact 
complex $0 \rightarrow(P, Y) \rightarrow E_{0} \rightarrow E_{1} \rightarrow \cdots$, with $E_{i}$ injective in $\bmod \Gamma$ for all $i \geq 0$. For $X$ in $\bmod \Lambda$ we get maps of complexes

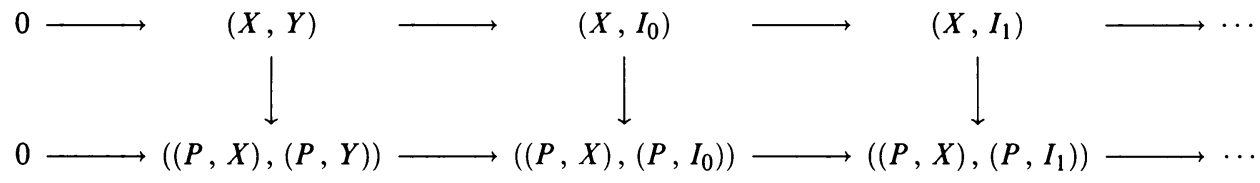

and

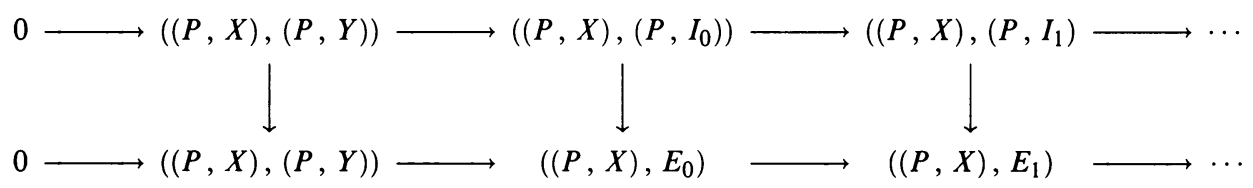

The composition of these maps induces a map $\rho_{X, Y}$ between the homologies of the involved complexes. This is, a family of maps $\rho_{X, Y}^{i}: \operatorname{Ext}_{\Lambda}^{i}(X, Y) \rightarrow$ $\operatorname{Ext}_{\Gamma}^{i}((P, X)(P, Y)), i \geq 0$, with $\rho_{X, Y}^{0}=\rho_{X, Y}$.

The following result relates extensions in $\bmod \Gamma$ and in $\bmod \Lambda$.

Theorem 3.2. Let $n \geq 0$. Then the map

$$
\rho_{X, Y}^{n}: \operatorname{Ext}_{\Lambda}^{n}(X, Y) \rightarrow \operatorname{Ext}_{\Gamma}^{n}((P, X),(P, Y))
$$

above defined is an isomorphism provided one of the three following conditions holds:

(a) $\mathbf{X}$ is in $\mathbf{P}_{i}, Y$ is in $\mathbf{I}_{j}$ and $n \leq i+j$.

(b) $X$ is in $\bmod \Lambda, Y$ in $\mathbf{I}_{n+1}$.

(c) $X$ is in $\mathbf{P}_{n+1}, Y$ in $\bmod \Lambda$.

Proof. In $n=0$ the theorem states the same as Lemma 3.1,2, (ii) and (iii). So we assume $n \geq 1$ and prove the theorem by induction on $n$. Let $X$ in $\mathbf{P}_{0}$ and consider an exact sequence $0 \rightarrow K \rightarrow P_{0} \rightarrow X \rightarrow 0$ with $P_{0}$ is add $P$. Then $0 \rightarrow(P, K) \rightarrow\left(P, P_{0}\right) \rightarrow(P, X) \rightarrow 0$ is exact and $\left(P, P_{0}\right)$ is projective in $\bmod \Gamma$. We get a diagram

$$
\begin{aligned}
& 0 \rightarrow(X, Y) \quad \rightarrow \quad\left(P_{0}, Y\right) \quad \rightarrow \\
& \begin{array}{l}
\rightarrow \\
\quad((P, X),(P, Y))
\end{array} \rightarrow\left(\left(P, \stackrel{\downarrow}{\rho_{X}, Y}\right),(P, Y)\right) \rightarrow
\end{aligned}
$$

$$
\begin{aligned}
& \begin{array}{c}
(K, Y) \\
\downarrow \rho_{K, Y}
\end{array} \quad \rightarrow \quad \begin{array}{c}
\operatorname{Ext}_{\Lambda}^{1}(X, Y) \\
\downarrow \rho_{X, Y}^{1}
\end{array} \quad \rightarrow \quad 0 \\
& ((P, K),(P, Y)) \rightarrow \operatorname{Ext}_{\Lambda}^{1}((P, X),(P, Y)) \rightarrow 0 .
\end{aligned}
$$

If $X$ is in $\mathbf{P}_{2}$ then $K$ is in $\mathbf{P}_{1}$. So it follows from Lemma 3.1, (2) (iii) that the three left vertical maps are isomorphisms if either $X$ is in $\mathbf{P}_{2}$ or $Y$ is in $\mathbf{I}_{1}$. Thus $\rho_{X, Y}^{1}$ is an isomorphism for $X$ in $\mathbf{P}_{2}, Y$ in $\mathbf{I}_{1}$. Considering an exact sequence $0 \rightarrow Y \rightarrow I_{0} \rightarrow I \rightarrow 0$ with $I_{0}$ in add $I$ one proves that $\rho_{X, Y}^{1}$ is an isomorphism if either $X \in \mathbf{P}_{1}$ or $Y \in \mathbf{I}_{2}$. Thus the theorem holds for $n=1$. 
Assume now that $n>1$. First we consider the case when $X$ is in $\mathbf{P}_{i}$ and $i \geq 0$. Let $0 \rightarrow K \rightarrow P_{0} \rightarrow X \rightarrow 0$ be an exact sequence with $P_{0}$ in add $P$. Then the sequence $0 \rightarrow(P, K) \rightarrow\left(P, P_{0}\right) \rightarrow(P, X) \rightarrow 0$ is exact, and since $X$ is in $\mathbf{P}_{0}$ we have that $\left(P, P_{0}\right)$ is projective in $\bmod \Gamma$. We get a commutative diagram

$$
\begin{array}{ccc}
\operatorname{Ext}_{\Lambda}^{n-1}(K, Y) & \longrightarrow & \operatorname{Ext}_{\Lambda}^{n}(X, Y) \\
\downarrow \rho_{L, Y}^{n-1} & & \downarrow_{X, Y}^{n} \\
\operatorname{Ext}_{\Gamma}^{n-1}((P, K),(P, Y)) & \longrightarrow \operatorname{Ext}_{\Gamma}^{n}((P, X),(P, Y))
\end{array}
$$

where the horizontal maps are isomorphisms. Since $X$ is in $\mathbf{P}_{i}$ and $i \geq 0$, then $K$ is in $\mathbf{P}_{i-1}$. Let now $Y$ be in $\mathbf{I}_{j}$ with $i+j=n$. We can apply the induction hypothesis to $K$ and $Y$ and conclude that $\rho_{K, Y}^{n-1}$ is an isomorphism. Thus $\rho_{X, Y}^{n}$ is also an isomorphism. If $Y$ is any $\Lambda$-module and $i=n+1$ the same argument holds.

The other cases follow in a similar way, considering an exact sequence $0 \rightarrow$ $Y \rightarrow I_{0} \rightarrow K \rightarrow 0$ with $I_{0}$ in $\operatorname{add} I$.

We observe that when $X$ is in $\mathbf{P}_{\infty}$ the theorem states that $\rho_{X, Y}^{n}$ : $\operatorname{Ext}_{\Lambda}^{n}(X, Y) \rightarrow \operatorname{Ext}_{\Gamma}^{n}((P, X),(P, Y))$ is an isomorphism for all $n \geq 0$ and for all $Y$ in $\bmod \Lambda$. Similarly, if $Y$ is in $\mathbf{I}_{\infty}$ then $\rho_{X, Y}^{n}$ is an isomorphism for all $n \geq 0$ and all $X$ in $\bmod \Lambda$. Since $(P):, \bmod \Lambda \rightarrow \bmod \Gamma$ is a dense functor we obtain the following corollaries.

Corollary 3.3. (a) If $X$ is in $\mathbf{P}_{\infty}$ then $p \operatorname{dim}_{\Lambda} X=p \operatorname{dim}_{\Gamma}(P, X)$.

(b) If $X$ is in $\mathbf{I}_{\infty}$ then inj $\operatorname{dim}_{\Lambda} X=\operatorname{inj} \operatorname{dim}_{\Gamma}(P, X)$.

Corollary 3.4. If $\mathbf{P}_{1}=\mathbf{P}_{\infty}$ or $\mathbf{I}_{1}=\mathbf{I}_{\infty}$ then $\operatorname{gl} \cdot \operatorname{dim} \Gamma \leq \operatorname{gl} \cdot \operatorname{dim} \Lambda$.

We observe that in general it is not true that $\operatorname{gl} \operatorname{dim} \Gamma \leq \operatorname{gl} \cdot \operatorname{dim} \Lambda$, as the following example shows.

Example 3.5. Let $k$ be any field and $\Lambda$ the $k$-algebra associated to the quiver

$$
\text { - } \underset{\beta}{\stackrel{\alpha}{\longrightarrow}} \text { ? }
$$

modulo the relation $\alpha \beta=0$. Let $P_{1}$ be the projective associated to the vertex 1. Then $\mathrm{gl} \cdot \operatorname{dim}\left(\operatorname{End}_{\Lambda}\left(P_{1}\right)\right)^{\mathrm{op}}=\infty$ and $\mathrm{gl} \cdot \operatorname{dim} \Lambda=2$.

Assume now that $Y$ is in $\mathbf{I}_{k}$ for some $0 \leq k \leq \infty$ and let $0 \rightarrow Y \rightarrow I_{0} \rightarrow$ $\cdots \rightarrow I_{k} \rightarrow \cdots$ be a minimal injective coresolution of $Y$. Then $I_{j}$ is in add $I$ for $j \leq k$, so that $0 \rightarrow(P, Y) \rightarrow\left(P, I_{0}\right) \rightarrow \cdots \rightarrow\left(P, I_{k}\right)$ is the beginning of an injective coresolution of $(P, Y)$. In particular, when $\mathbf{I}_{1}=\mathbf{I}_{\infty}$ the injective coresolutions of the $\Gamma$-modules are all obtained by applying the functor $(P, \quad)$ to injective coresolutions of $\Lambda$-modules. So we want to know what modules are in $\mathbf{I}_{k}$, and give necessary and sufficient conditions for $\mathbf{I}_{1}$ to be equal to $\mathbf{I}_{\infty}$. We are interested also in dual results about $\mathbf{P}_{k}$ to study projective resolutions.

Using the preceding theorem we can give the following characterization of modules in $\mathbf{I}_{k}$. 
Theorem 3.6. Let $1 \leq k \leq \infty$. Then

(a) $A \Lambda$-module $\bar{Y}$ is in $\mathbf{I}_{k}$ if and only if the above defined map $\rho_{X, Y}^{i}$ : $\operatorname{Ext}_{\Lambda}^{i}(X, Y) \rightarrow \operatorname{Ext}_{\Gamma}^{i}((P, X),(P, Y))$ is an isomorphism, for all $0 \leq i \leq k-1$ and all $X$ in $\bmod \Lambda$.

(b) $A \Lambda$-module $X$ is in $\mathbf{P}_{k}$ if and only if

$$
\rho_{X, Y}^{i}: \operatorname{Ext}_{\Lambda}^{i}(X, Y) \rightarrow \operatorname{Ext}_{\Gamma}^{i}((P, X),(P, Y))
$$

is an isomorphism, for all $0 \leq i \leq k-1$ and all $Y$ in $\bmod \Lambda$.

Proof. (a) The fact that $Y$ in $\mathbf{I}_{k}$ implies that

$$
\operatorname{Ext}_{\Lambda}^{i}(X, Y) \simeq \operatorname{Ext}_{\Gamma}^{i}((P, X),(P, Y))
$$

for all $X$ in $\bmod \Lambda$ and all $0 \leq i \leq k-1$ is the statement (b) of Theorem 3.2. To prove the converse we will use the characterization of modules in $\mathbf{I}_{k}$ given in Proposition 2.6, stating that a $\Lambda$-module $M$ is in $\mathbf{I}_{k}$ if and only if $\operatorname{Ext}_{\Lambda}^{i}(\Lambda / \mathfrak{A}, M)=0$ for $i=0, \ldots, k$.

Let $i \geq 0$. We consider the commutative diagram

$$
\begin{array}{cc}
\operatorname{Ext}_{\Lambda}^{i}(\Lambda, Y) & \operatorname{Ext}_{\Lambda}^{i}(\mathfrak{A}, Y) \\
\downarrow \rho_{\Lambda, Y}^{i} & \\
\operatorname{Ext}_{\Gamma}^{i}((P, \Lambda),(P, Y)) & \longrightarrow \rho_{\mathfrak{A}, Y}^{i} \\
& \longrightarrow \operatorname{Ext}_{\Gamma}^{i}((P, \mathfrak{A}),(P, Y))
\end{array}
$$

induced by the inclusion $\mathfrak{A} \rightarrow \Lambda$. The lower horizontal map is an isomorphism because $(P, \mathfrak{A}) \rightarrow(P, \Lambda)$ is an isomorphism.

We assume now that $\rho_{X, Y}^{i}: \operatorname{Ext}_{\Lambda}^{i}(X, Y) \rightarrow \operatorname{Ext}_{\Gamma}^{i}((P, X),(P, Y))$ is an isomorphism for all $0 \leq i \leq k-1$ and all $X$ in $\bmod \Lambda$. Then the vertical maps in the diagram are isomorphisms and therefore $\operatorname{Ext}_{\Lambda}^{i}(\Lambda, Y) \simeq \operatorname{Ext}_{\Lambda}^{i}(\mathfrak{A}, Y)$, for $0 \leq i \leq k-1$. Hence $\operatorname{Ext}_{\Lambda}^{i}(\mathfrak{A}, Y)=0$ for $1 \leq i \leq k-1$. On the other hand, from the exact sequence $0 \rightarrow \mathfrak{A} \rightarrow \Lambda \rightarrow \Lambda / \mathfrak{A} \rightarrow 0$ we get an exact sequence $0 \rightarrow(\Lambda / \mathfrak{A}, Y) \rightarrow(\Lambda, Y) \rightarrow(\mathfrak{A}, Y) \rightarrow \operatorname{Ext}_{\Lambda}^{1}(\Lambda / \mathfrak{A}, Y) \rightarrow 0$. We just proved that $(\Lambda, Y) \rightarrow(\mathfrak{A}, Y)$ is an isomorphism, so $(\Lambda / \mathfrak{A}, Y)=0$ and $\operatorname{Ext}_{\Lambda}^{1}(\Lambda / \mathfrak{A}, Y)=0$. Thus $\operatorname{Ext}_{\Lambda}^{1}(\Lambda / \mathfrak{A}, Y)=0$ for all $0 \leq i \leq k$, this is, $Y$ is in $\mathbf{I}_{k}$.

(b) follows from (a) by duality.

The following propositions gives another characterization of modules in $\mathbf{I}_{k}$.

Proposition 3.7. (a) Let $X$ be in $\mathbf{I}_{1}$ and $k \geq 1$. Then $X$ is in $\mathbf{I}_{k}$ if and only if $\operatorname{Ext}_{\Gamma}^{i}\left(P^{*},(P, X)\right)=0$ for all $1 \leq i \leq k-1$, where $P^{*}$ denotes the $\Gamma$-module $\operatorname{Hom}_{\Lambda}(P, \Lambda)$.

(b) Let $X$ be in $\mathbf{P}_{1}$ and $k \geq 1$. Then $X$ is in $\mathbf{P}_{k}$ if and only if

$$
\operatorname{Tor}_{i}^{\Gamma}(P,(P, X))=0
$$

for all $1 \leq i \leq k-1$, where $P$ is considered as a module over $\Gamma^{\mathrm{op}}=\operatorname{End}_{\Lambda}(P)$ in the natural way.

Proof. (a) Let $X$ be in $\mathbf{I}_{k}$. Then we know by Proposition 3.6 (a) that

$$
\operatorname{Ext}_{\Gamma}^{i}\left(P^{*},(P, X)\right)=\operatorname{Ext}_{\Gamma}^{i}((P, \Lambda),(P, X)) \simeq \operatorname{Ext}_{\Lambda}^{i}(\Lambda, X),
$$

for $0 \leq i \leq k-1$. This proves that $\operatorname{Ext}_{\Gamma}^{i}\left(P^{*},(P, X)\right)=0$ for $1 \leq i \leq k-1$. 
To prove the converse we assume that $\operatorname{Ext}_{\Gamma}^{i}\left(P^{*},(P, X)\right)=0$ for $1 \leq i \leq$ $k-1$. We will prove that $X$ is in $\mathbf{I}_{k}$ by induction on $k$. The result is true for $k=1$. So let $k>1$. Then $X$ is in $\mathbf{I}_{1}$, and since $\mathfrak{A}$ is in $\mathbf{P}_{0}$ we can apply Theorem 3.2 and obtain that $\operatorname{Ext}_{\Gamma}^{1}((P, \mathfrak{A}),(P, X)) \simeq \operatorname{Ext}_{\Lambda}^{1}(\mathfrak{A}, X)$. Since $(P, \mathfrak{A}) \simeq P^{*}$ and we are assuming that $\operatorname{Ext}_{\Gamma}^{i}\left(P^{*},(P, X)\right)=0$ for $i \leq k-1$ we obtain that $\operatorname{Ext}_{\Lambda}^{2}(\Lambda / \mathfrak{A}, X) \simeq \operatorname{Ext}_{\Lambda}^{1}(\mathfrak{A}, X)=0$. So $X \in \mathbf{I}_{2}$.

Consider now an exact sequence $0 \rightarrow X \rightarrow I_{0} \rightarrow L \rightarrow 0$ with $I_{0}$ in add $I$. Then $0 \rightarrow(P, X) \rightarrow\left(P, I_{0}\right) \rightarrow(P, L) \rightarrow 0$ is exact and $\left(P, I_{0}\right)$ is an injective $\Gamma$-module. So $\operatorname{Ext}_{\Gamma}^{i}\left(P^{*},(P, L)\right)=0$ for $1 \leq i \leq k-2$. Since $X$ is in $I_{2}$ it follows that $L$ is in $I_{1}$, so the induction hypothesis applies to $L$. Therefore $L$ is in $\mathbf{I}_{k-1}$, so $X$ is in $\mathbf{I}_{k}$, as we wanted to prove.

(b) follows from (a) by duality, observing that $\mathfrak{A}=\tau_{P^{*}}\left(\Lambda^{\text {op }}\right)$ as is also $\tau_{P}(\Lambda)$.

We characterize now when $\mathbf{I}_{1}=\mathbf{I}_{\infty}$, and when $\mathbf{P}_{1}=\mathbf{P}_{\infty}$.

Corollary 3.8. (a) $\mathbf{I}_{1}=\mathbf{I}_{\infty}$ if and only if the $\Gamma$-module $P^{*}$ is projective.

(b) $\mathbf{P}_{1}=\mathbf{P}_{\infty}$ if and only if the $\Gamma^{\mathrm{op}}$-module $P$ is projective.

Proof. (a) follows directly from the Proposition 3.7. To prove (b) we write $\mathfrak{A}=\tau_{P^{*}}\left(\Lambda^{\mathrm{op}}\right)$. Since $\mathbf{P}_{1}=\mathbf{P}_{\infty}$ we get that the subcategories $\mathbf{I}_{1, P^{*}}$ and $\mathbf{I}_{\infty, P^{*}}$ of $\bmod \Lambda^{\mathrm{op}}$ are the same. By (a) we obtain that the $\Gamma^{\mathrm{op}}$-module $\left(P^{*}\right)^{*}=P$ is projective.

We recall now some properties of the functor $\operatorname{Hom}_{\Gamma}\left(P^{*},\right)=\left(P^{*},\right): \bmod \Gamma$ $\rightarrow \bmod \Lambda$, which is the right adjoint of $(P):, \bmod \Lambda \rightarrow \bmod \Gamma[A, \S 5]$. The functor $\left(P^{*},\right)$ is left exact, fully faithful and its image is the subcategory $\mathbf{I}_{1}$ of $\bmod \Lambda$. The composition $\left.\bmod \Gamma \stackrel{\left(P^{*},\right.}{\longrightarrow}\right) \bmod \Lambda \stackrel{(P,)}{\longrightarrow} \bmod \Gamma$ is the identity of $\bmod \Gamma$, and $\left(P^{*},\right)$ preserves injective envelopes. However, $\left(P^{*},\right)$ does not preserve injective coresolutions, not even injective copresentations. In fact, given an injective coresolution $0 \rightarrow M \rightarrow I_{0} \rightarrow I_{1} \rightarrow \cdots$ of a $\Gamma$-module $M$, then $0 \rightarrow\left(P^{*}, M\right) \rightarrow\left(P^{*}, I_{0}\right) \rightarrow \cdots \rightarrow\left(P^{*}, I_{k}\right)$ is the beginning of an injective coresolution of $\left(P^{*}, M\right)$ if and only if $\operatorname{Ext}_{\Gamma}^{i}\left(P^{*}, M\right)=0$ for $i=1, \ldots, k$. Therefore Proposition 3.7(a) can now be written in the following way.

Proposition 3.9. Let $X$ be a $\Lambda$-module and $M=(P, X)$. Then the following conditions are equivalent for $1 \leq k \leq \infty$.

(i) $X$ is in $\mathbf{I}_{k}$.

(ii) If $0 \rightarrow M \rightarrow I_{0} \rightarrow I_{1} \rightarrow \cdots$ is an injective resolution of $M$ in $\bmod \Gamma$ then $0 \rightarrow X \rightarrow\left(P^{*}, I_{0}\right) \rightarrow \cdots \rightarrow\left(P^{*}, I_{k-1}\right)$ is the beginning of an injective coresolution of $X=\left(P^{*}, M\right)$ in $\bmod \Lambda$.

It follows that $\mathbf{I}_{1}=\mathbf{I}_{\infty}$ if and only if the functor $\left(P^{*},\right): \bmod \Gamma \rightarrow \bmod \Lambda$ carries injective coresolution of $\Lambda$-modules into injective coresolutions of $\Lambda$ modules.

On the other hand, $(P):, \bmod \Lambda \rightarrow \bmod \Gamma$ has also a left adjoint, the functor $P \otimes_{\Gamma}: \bmod \Gamma \rightarrow \bmod \Lambda[\mathrm{A}, \S 5]$. This functor is right exact, fully faithful and its image is $\mathbf{P}_{1}$. The composition 


$$
\bmod \Gamma \stackrel{P \otimes_{\Gamma}}{\longrightarrow} \bmod \Lambda \stackrel{(P,)}{\longrightarrow} \bmod \Gamma
$$

is the identity of $\bmod \Gamma$, and $P \otimes_{\Gamma}$ preserves projective covers. Proposition 3.7(b) can be written as follows.

Proposition 3.10. Let $X$ be a $\Lambda$-module and $M=(P, X)$. Then the following conditions are equivalent for $1 \leq k \leq \infty$.

(i) $X$ is in $\mathbf{P}_{k}$.

(ii) If $\cdots \rightarrow P_{k} \rightarrow \cdots \rightarrow P_{0} \rightarrow M \rightarrow 0$ is a projective resolution of $M$ in $\bmod \Gamma$ then $P \otimes_{\Gamma} P_{k} \rightarrow \cdots \rightarrow P \otimes_{\Gamma} P_{0} \rightarrow X \rightarrow 0$ is the beginning of a projective resolution of $X=P \otimes_{\Gamma} M$ in $\bmod \Lambda$.

\section{CONDITIONS FOR $\mathbf{I}_{1}=\mathbf{I}_{\infty}$}

In this section we give other necessary and sufficient conditions for $\mathbf{I}_{1}$ to be equal to $\mathbf{I}_{\infty}$, and for $\mathbf{P}_{1}$ to be equal to $\mathbf{P}_{\infty}$. We prove that $\mathbf{I}_{1}=\mathbf{I}_{\infty}$ if and only if $\mathfrak{A} \otimes_{\Lambda} \mathfrak{A}$ is a projective $\Lambda$-module, and $\mathbf{P}_{1}=\mathbf{P}_{\infty}$ if and only if $\mathfrak{A} \otimes_{\Lambda} \mathfrak{A}$ is a projective $\Lambda^{\mathrm{op}}$-module.

We start by recalling some further properties of the functor $P \otimes_{\Gamma}: \bmod \Gamma \rightarrow$ $\bmod \Lambda$. (See, for example, $[A, \S 5]$.) Since $P \otimes_{\Gamma}: \bmod \Gamma \rightarrow \bmod \Lambda$ is a left adjoint of $(P):, \bmod \Lambda \rightarrow \bmod \Gamma$, there is a unique isomorphism of functors $f$ from the composition $\left(P \otimes_{\Gamma}\right)(P, \quad)$ to the identity of $\bmod \Lambda$ such that $(P)$,$f is an isomorphism. For X$ in $\bmod \Lambda, f_{X}: P \otimes_{\Gamma}(P, X) \rightarrow X$ is given by $f_{X}(p \otimes h)=h(p)$, for $h \in(P, X), p \in P$. The following is a well-known fact.

Lemma 4.1. Let $X$ be in $\bmod \Lambda$ and $f_{X}: P \otimes_{\Gamma}(P, X) \rightarrow X$ be as above. Then

(1) $P \otimes_{\Gamma}(P, X)$ is in $\mathbf{P}_{1}$ and $\operatorname{ker} f_{X}, \operatorname{Coker} f_{X}$ are in $\bmod \Lambda / \mathfrak{A}$.

(2) Given an exact sequence $0 \rightarrow L_{1} \rightarrow X_{1} \stackrel{\varphi}{\longrightarrow} X \rightarrow L_{2} \rightarrow 0$ with $X_{1}$ in $\mathbf{P}_{1}$ and $L_{1}, L_{2}$ in $\bmod \Lambda / \mathfrak{A}$ we have:

(a) There is a commutative diagram

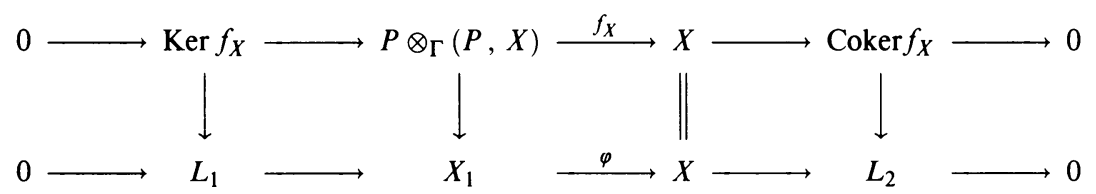

where the vertical arrows are isomorphisms.

(b) $(P, \varphi):\left(P, X_{1}\right) \rightarrow(P, X)$ is an isomorphism.

(c) Let $Y$ in $\mathbf{P}_{k}$ and $1 \leq k \leq \infty$. The maps $\operatorname{Ext}_{\Lambda}^{i}(Y, \varphi): \operatorname{Ext}_{\Lambda}^{i}\left(Y, X_{1}\right) \rightarrow$ $\operatorname{Ext}_{\Lambda}^{i}(Y, X)$ are isomorphisms for all $0 \leq i \leq k-1$.

Now we state the dual results (see, for example, $[A, \S 5])$. Since $\left(P^{*},\right): \bmod \Gamma$ $\rightarrow \bmod \Lambda$ is a right adjoint of $(P):, \bmod \Lambda \rightarrow \bmod \Gamma$ there is a unique isomorphism of functors $g$ from the identity of $\bmod \Lambda$ to the composition $\left(P^{*},\right)(P,) \simeq\left(P \otimes_{\Gamma} P^{*},\right)$. For $X$ in $\bmod \Lambda, g_{X}: X \rightarrow\left(P^{*},(P, X)\right)$ is given by $\left(g_{X}(x)(f)\right)(p)=f(p) x$, for $x$ in $X, f$ in $P^{*}$ and $p$ in $P$.

Lemma 4.2. Let $X$ in $\bmod \Lambda$ and $g_{X}: X \rightarrow\left(P^{*},(P, X)\right)$ be as above. Then

(1) $\left(P^{*},(P, X)\right)$ is in $\mathbf{I}_{1}$ and $\operatorname{Ker} g_{X}$, Coker $g_{X}$ are in $\bmod \Lambda / \mathfrak{A}$.

(2) Given an exact sequence $0 \rightarrow L_{1} \rightarrow X \stackrel{\psi}{\longrightarrow} X_{1} \rightarrow L_{2} \rightarrow 0$ with $X_{1}$ in $\mathbf{I}_{1}$ and $L_{1}, L_{2}$ in $\bmod \Lambda / \mathfrak{A}$, we have: 
(a) There is a commutative diagram

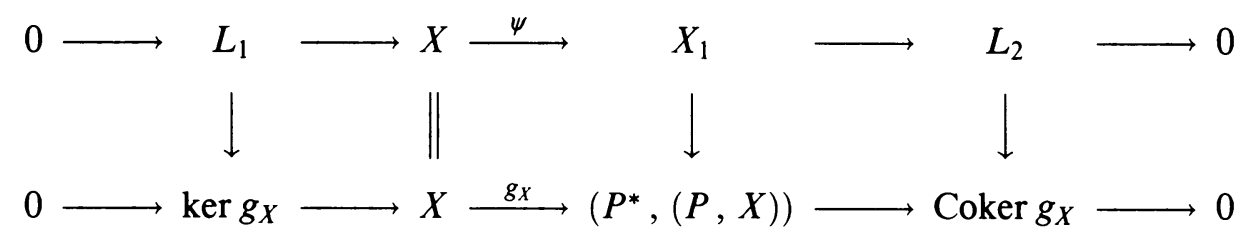

where the vertical arrows are isomorphisms.

(b) $(P, \psi):(P, X) \rightarrow\left(P, X_{1}\right)$ is an isomorphism.

(c) Let $Y$ in $\mathbf{I}_{k}$ and $1 \leq k \leq \infty$. The maps $\operatorname{Ext}_{\Lambda}^{i}(\psi, Y)$ : $\operatorname{Ext}_{\Lambda}^{i}\left(X_{1}, Y\right) \rightarrow$ $\operatorname{Ext}_{\Lambda}^{i}(X, Y)$ are isomorphisms for all $0 \leq i \leq k-1$.

To simplify notation we denote $P \otimes_{\Gamma}(P, X)$ by $X_{P_{1}}$, and $\left(P^{*},(P, X)\right)$ by $X_{I_{1}}$.

In the following proposition we describe $\Lambda_{P_{1}}$.

Proposition 4.3. There are isomorphisms of $\Lambda$-modules $\Lambda_{P_{1}} \simeq \mathfrak{A}_{P_{1}} \simeq \mathfrak{A} \otimes_{\Lambda} \mathfrak{A}$.

Proof. To prove the result we will use the following lemma.

Lemma 4.4. Let $P_{0}$ be the projective cover of $\mathfrak{A}$. Then $P_{0}$ is also the projective cover of $\mathfrak{A} \otimes_{\Lambda} \mathfrak{A}$, and there is an exact sequence

$$
0 \rightarrow \operatorname{Tor}_{3}^{\Lambda}(\Lambda / \mathfrak{A}, \Lambda / \mathfrak{A}) \rightarrow C \rightarrow P_{0} \rightarrow \mathfrak{A} \otimes_{\Lambda} \mathfrak{A} \rightarrow 0
$$

with $C$ in $\mathbf{P}_{0}$. So $\mathfrak{A} \otimes_{\Lambda} \mathfrak{A}$ is in $\mathbf{P}_{1}$.

Proof. Consider the exact sequence $0 \rightarrow K \rightarrow P_{0} \rightarrow \mathfrak{A} \rightarrow 0$. Tensoring with $\mathfrak{A}$ we obtain the exact sequence

$$
0 \rightarrow \operatorname{Tor}_{1}^{\Lambda}(\mathfrak{A}, \mathfrak{A}) \rightarrow \mathfrak{A} \otimes K \rightarrow \mathfrak{A} \otimes P_{0} \rightarrow \mathfrak{A} \otimes_{\Lambda} \mathfrak{A} \rightarrow 0 .
$$

On the other hand, we have a commutative diagram

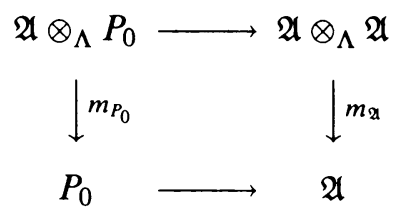

where the vertical maps are the multiplication maps. We know that $\mathfrak{A} P_{0}=P_{0}$ because $P_{0}$ is in add $P$. So, tensoring the exact sequence $0 \rightarrow \mathfrak{A} \rightarrow \Lambda \rightarrow$ $\Lambda / \mathfrak{A} \rightarrow 0$ with the projective module ${ }_{\Lambda} P_{0}$ we obtain that $\mathfrak{A} \otimes_{\Lambda} P_{0} \rightarrow \Lambda \otimes_{\Lambda} P_{0}$ is an isomorphism. Thus $m_{P_{0}}$ is an isomorphism. From the commutativity of the diagram it follows that $P_{0}$ is the projective cover of $\mathfrak{A} \otimes_{\Lambda} \mathfrak{A}$. This ends the proof of the lemma, since $\operatorname{Tor}_{1}^{\wedge}(\mathfrak{A}, \mathfrak{A}) \simeq \operatorname{Tor}_{3}^{\Lambda}(\Lambda / \mathfrak{A}, \Lambda / \mathfrak{A})$, and $C=\mathfrak{A} \otimes_{\Lambda} K$ is in $\mathbf{P}_{0}$ because $\mathfrak{A} C=C$.

Now we prove the proposition. We have that $(P, \Lambda) \simeq\left(P, \tau_{P} \Lambda\right)=(P, \mathfrak{A})$. Thus $\left(P, \Lambda_{P_{1}}\right) \simeq\left(P, \mathfrak{A}_{P_{1}}\right)$, as follows from Lemma 4.1.2(b). Since both $\Lambda_{P}$, $\mathfrak{A}_{P_{1}}$ are in $\mathbf{P}_{1}$ and the restriction of $(P$,$) to \mathbf{P}_{1}$ is an equivalence we get that $\Lambda_{P_{1}} \simeq \mathfrak{A}_{P_{1}}$.

Consider the exact sequence $0 \rightarrow \operatorname{Tor}_{2}^{\Lambda}(\Lambda / \mathfrak{A}, \Lambda / \mathfrak{A}) \rightarrow \mathfrak{A} \otimes_{\Lambda} \mathfrak{A} \rightarrow \mathfrak{A} \rightarrow 0$, as in Lemma 1.5. The module $\operatorname{Tor}_{2}^{\Lambda}(\Lambda / \mathfrak{A}, \Lambda / \mathfrak{A})$ is in $\bmod \Lambda / \mathfrak{A}$, and $\mathfrak{A} \otimes_{\Lambda} \mathfrak{A}$ is in $\mathbf{P}_{1}$, by the lemma. The uniqueness of a sequence $0 \rightarrow L_{1} \rightarrow X_{1} \rightarrow \mathfrak{A} \rightarrow$ 
$L_{2} \rightarrow 0$ with $X_{1}$ in $\mathbf{P}_{1}$ and $L_{1}, L_{2}$ in $\bmod \Lambda / \mathfrak{A}$ (Lemma 4.1) implies that $\mathfrak{A}_{P_{1}} \simeq \mathfrak{A} \otimes_{\Lambda} \mathfrak{A}$.

We can now describe when $\mathfrak{A} \otimes_{\Lambda} \mathfrak{A}$ is a projective $\Lambda$-module.

Proposition 4.5. The following are equivalent for the idempotent ideal $\mathfrak{A}$

(a) $\mathbf{I}_{1}=\mathbf{I}_{\infty}$.

(b) $\mathfrak{A} \otimes_{\Lambda} \mathfrak{A}$ is a projective $\Lambda$-module.

(c) $\mathfrak{A} \otimes_{\Lambda} \mathfrak{A}$ is a projective $\Lambda$-module and $\operatorname{Tor}_{3}^{\Lambda}(\Lambda / \mathfrak{A}, \Lambda / \mathfrak{A})=0$.

Proof. We proved in Corollary 3.8 that $\mathbf{I}_{1}=\mathbf{I}_{\infty}$ if and only if $P^{*}$ is a projective $\Gamma$-module. This is the case if and only if $P^{*}=(P, Q)$ for some $Q$ in add $P$, as follows from Lemma 3.1,3. On the other hand $\left.(P)\right|_{,\mathbf{P}_{1}}: \mathbf{P}_{1} \rightarrow \bmod \Gamma$ is an equivalence of categories and $P^{*}=(P, \Lambda)=\left(P, \Lambda_{P_{1}}\right)$. So $P^{*}$ is a projective $\Gamma$-module if and only if $\Lambda_{P_{1}}$ is a projective $\Lambda$-module. Therefore (a) $\Leftrightarrow(\mathrm{b})$, since we have proven in Proposition 4.3 that $\Lambda_{P_{1}}$ and $\mathfrak{A} \otimes \mathfrak{A}$ are isomorphic $\Lambda$-modules.

Clearly $(\mathrm{c}) \Rightarrow(\mathrm{b})$. We prove now $(\mathrm{b}) \Rightarrow(\mathrm{c})$. We consider the exact sequence $0 \rightarrow \operatorname{Tor}_{\mathfrak{3}}^{\Lambda}(\Lambda / \mathfrak{A}, \Lambda / \mathfrak{A}) \rightarrow C \rightarrow P_{0} \rightarrow \mathfrak{A} \otimes \mathfrak{A} \rightarrow 0$ with $C$ in $\mathbf{P}_{0}$ and $P_{0}=$ $P_{0}(\mathfrak{A} \otimes \mathfrak{A})$, as in Lemma 4.4. If $\mathfrak{A} \otimes \mathfrak{A}$ is projective then $\operatorname{Tor}_{3}^{\Lambda}(\Lambda / \mathfrak{A}, \Lambda / \mathfrak{A}) \rightarrow C$ is an isomorphism. From this it follows that $C=0$. Because on one hand $\mathfrak{A} C=C$ since $C$ is in $\mathbf{P}_{0}$, and on the other hand $C \simeq \operatorname{Tor}_{3}^{\Lambda}(\Lambda / \mathfrak{A}, \Lambda / \mathfrak{A})$ is a $\Lambda / \mathfrak{A}$ module.

By the definition of $\Lambda_{P_{1}}$ we know that $\Lambda_{P_{1}}=P \otimes_{\Gamma}(P, \Lambda) \simeq P \otimes_{\Gamma} P^{*}$. On the other hand Proposition 4.3 shows that $\Lambda_{P_{1}} \simeq \mathfrak{A} \otimes_{\Lambda} \mathfrak{A}$. Therefore $P \otimes_{\Gamma} P^{*}$ and $\mathfrak{A} \otimes_{\Lambda} \mathfrak{A}$ are isomorphic as left $\Lambda$-modules. We show next that they are, in fact, isomorphic $\Lambda$ - $\Lambda$-bimodules by giving a bimodule isomorphism $t: P \otimes_{\Gamma} P^{*} \rightarrow$ $\mathfrak{A} \otimes_{\Lambda} \mathfrak{A}$.

Proposition 4.6. There is a $\Lambda$ - $\Lambda$-bimodule isomorphism $t: P \otimes_{\Gamma} P^{*} \rightarrow \mathfrak{A} \otimes_{\Lambda} \mathfrak{A}$ such that the diagram

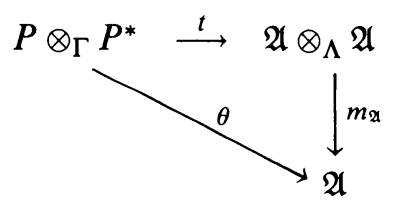

commutes where $m_{\mathfrak{A}}$ is the multiplication map, and $\theta(p \otimes f)=f(p)$, for $p \in P$, $f \in P^{*}$.

Proof. Let $\varepsilon: P^{*} \otimes_{\Lambda} P \rightarrow \Gamma$ be defined by $\varepsilon(f \otimes p)\left(p^{\prime}\right)=f\left(p^{\prime}\right) p$, for $f \in$ $P^{*}, p, p^{\prime} \in P$. Then $\varepsilon$ is a $\Lambda$ - $\Lambda$-bimodule isomorphism and induces an isomorphism $\alpha: P \otimes_{\Gamma}\left(P^{*} \otimes_{\Lambda} P\right) \otimes_{\Gamma} P^{*} \rightarrow P \otimes_{\Gamma} P^{*}$ of $\Lambda$ - $\Lambda$-bimodules. We get a commutative diagram

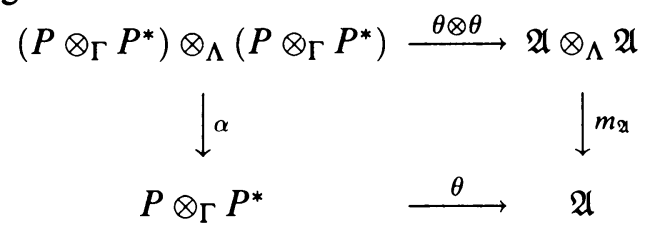

We observed just before the proposition that $P \otimes_{\Gamma} P^{*}$ and $\mathfrak{A} \otimes_{\Lambda} \mathfrak{A}$ are isomorphic in $\bmod \Lambda$. Thus the epimorphism $t=(\theta \otimes \theta) \alpha^{-1}: P \otimes_{\Gamma} P^{*} \rightarrow \mathfrak{A} \otimes_{\Lambda} \mathfrak{A}$ is an isomorphism and one easily checks that the required diagram commutes. 
Remark. We give an elementwise definition of $t$. Let $\operatorname{id}_{P}: P \rightarrow P$ be the identity map. Since $\varepsilon: P^{*} \otimes_{\Lambda} P \rightarrow \Gamma$ above defined is an isomorphism, there are $f_{i} \in P^{*}, p_{i} \in P, i=1, \ldots, n$, such that $\varepsilon\left(\sum_{i=1}^{n} f_{i} \otimes p_{i}\right)=\mathrm{id}_{P}$. Then $t(p \otimes f)=\sum_{i=1}^{n} f_{i}(p) \otimes f\left(p_{i}\right)$, for $p \in P, f \in P^{*}$.

As immediate consequences of the proposition we obtain the following two corollaries.

Corollary 4.7. The idempotent ideal $\mathfrak{A}$ is 2-idempotent if and only if the map $\theta: P \otimes_{\Gamma} P^{*} \rightarrow \mathfrak{A}$ defined above is an isomorphism.

Corollary 4.8. Let $X$ in $\bmod \Lambda$. Then $X_{I_{1}}=\left(\mathfrak{A} \otimes_{\Lambda} \mathfrak{A}, X\right)$ and $X_{P_{1}}=\mathfrak{A} \otimes_{\Lambda}$ $\mathfrak{A} \otimes_{\Lambda} X$. If $\mathfrak{A}$ is a 2-idempotent then $X_{I_{1}}=(\mathfrak{A}, X)$ and $X_{P_{1}}=\mathfrak{A} \otimes_{\Lambda} X$.

We know that if $\mathfrak{A}$ is an idempotent ideal then $\mathfrak{A} \otimes_{\Lambda} \mathfrak{A}$ is not isomorphic to $\mathfrak{A}$, unless $\mathfrak{A}$ is 2 -idempotent. However, we have the following result.

Corollary 4.9. The map $\mathrm{id}_{\mathfrak{A}} \otimes m_{\mathfrak{A}}: \mathfrak{A} \otimes_{\Lambda} \mathfrak{A} \otimes_{\Lambda} \mathfrak{A} \rightarrow \mathfrak{A} \otimes_{\Lambda} \mathfrak{A}$ is an isomorphism, where $\mathrm{id}_{\mathfrak{A}}: \mathfrak{A} \rightarrow \mathfrak{A}$ is the identity map and $m_{\mathfrak{A}}: \mathfrak{A} \otimes_{\Lambda} \mathfrak{A} \rightarrow \mathfrak{A}$ is the multiplication map.

Proof. We have a commutative diagram

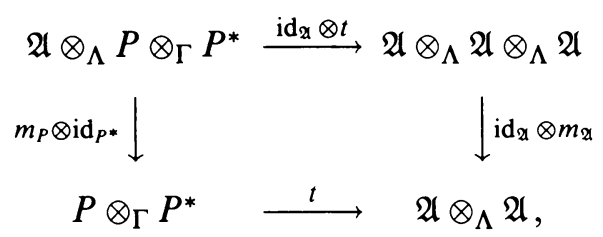

where $m_{P}: \mathfrak{A} \otimes_{\Lambda} P \rightarrow P$ is the multiplication map. Since both $m_{P}$ and $t$ are isomorphisms it follows that all the maps in the diagram are isomorphisms, proving the corollary.

We give now a list of necessary and sufficient conditions for $\mathbf{I}_{1}$ to be equal to $\mathbf{I}_{\infty}$, summarizing many of the previous results.

Proposition 4.10. The following are equivalent for an idempotent ideal $\mathfrak{A}$ of $\Lambda$ :

(1) $\mathbf{I}_{1}=\mathbf{I}_{\infty}$

(2) $\mathbf{I}_{1}=\mathbf{I}_{2}$.

(3) $P^{*}$ is a projective $\Gamma$-module.

(4) $\mathfrak{A} \otimes_{\Lambda} \mathfrak{A}$ is a projective $\Lambda$-module.

(5) $P \otimes_{\Gamma} P^{*}$ is a projective $\Lambda$-module.

(6) The composition $\bmod \Lambda \stackrel{(P,)}{\longrightarrow} \bmod \Gamma \stackrel{\left(P^{*}\right)}{\longrightarrow} \bmod \Lambda$ is exact.

It would be nice to know what modules are in $\mathbf{I}_{k}$ and not in $\mathbf{I}_{k+1}$ for a given idempotent ideal $\mathfrak{A}$. We observe that $\mathbf{I}_{k}=\mathbf{I}_{k+1}$ implies $\mathbf{I}_{k}=\mathbf{I}_{\infty}$. It would also be nice to know which is the smallest $k$ such that $\mathbf{I}_{k}=\mathbf{I}_{\infty}$, or, what is equivalent, such that $\mathbf{I}_{k}=\mathbf{I}_{k+1}$. We know that such $k$ is 1 if and only if $\mathfrak{A} \otimes_{\Lambda} \mathfrak{A}$ is projective. Moreover, the following result is true

Proposition 4.11. Let $\mathfrak{A}$ be an idempotent ideal and assume that

$$
\text { p.dim } \operatorname{dim}_{\Lambda}\left(\mathfrak{A} \otimes_{\Lambda} \mathfrak{A}\right)=r .
$$

Then $\mathbf{I}_{r+1}=\mathbf{I}_{r+2}=\cdots=\mathbf{I}_{\infty}$.

Proof. Assume $X$ is in $\mathbf{I}_{r+1}$. We know by Proposition 3.7 that this means that $\operatorname{Ext}_{\Gamma}^{i}\left(P^{*},(P, X)\right)=0$ for $i=1, \ldots, r$. To prove that $X$ is in $\mathbf{I}_{r+2}$ we will 
prove that $\operatorname{Ext}_{\Gamma}^{r+1}\left(P^{*},(P, X)\right)=0$. We write $P^{*}=(P, \Lambda)=\left(P, \Lambda_{P_{1}}\right)$. Since $\Lambda_{P_{1}}$ is in $\mathbf{P}_{1}$ and $X$ is in $\mathbf{I}_{r+1}$ it follows from Theorem 3.2 that

$$
\operatorname{Ext}_{\Gamma}^{r+1}\left(P^{*},(P, X)\right) \simeq \operatorname{Ext}_{\Lambda}^{r+1}\left(\Lambda_{P_{1}}, X\right) .
$$

And $\operatorname{Ext}_{\Lambda}^{r+1}\left(\Lambda_{P_{1}}, X\right)=0$ because we are assuming that $\operatorname{pdim}_{\Lambda}\left(\mathfrak{A} \otimes_{\Lambda} \mathfrak{A}\right)=r$, and $\Lambda_{P_{1}} \simeq \mathfrak{A} \otimes_{\Lambda} \mathfrak{A}$, by Proposition 4.3. So $\mathbf{I}_{r+1}=\mathbf{I}_{r+2}$.

Remark. The converse of Proposition 4.11 is not true. Let $\Lambda, \mathfrak{A}, P$ be as in Example $3, \S 1$. Then $\mathfrak{A}$ is a strong idempotent, so it is a 2 -idempotent and thus $\mathfrak{A} \otimes \mathfrak{A}=\mathfrak{A}$. However, $\mathbf{I}_{2}=\mathbf{I}_{\infty}$ and $\operatorname{p.dim} \mathfrak{A}=\infty$.

Using that $\mathfrak{A}=\tau_{P}(\Lambda)$ implies that $\mathfrak{A}=\tau_{P^{*}}\left(\Lambda^{\mathrm{op}}\right)$ we obtain from Proposition 4.10 the following result.

Proposition 4.12. The following conditions are equivalent for the idempoent ideal $\mathfrak{A}$ of $\Lambda$,

(1) $\mathbf{P}_{1}=\mathbf{P}_{\infty}$.

(2) $\mathbf{P}_{1}=\mathbf{P}_{2}$.

(3) $P$ is a projective $\Gamma^{\mathrm{op}}$-module.

(4) $\mathfrak{A} \otimes_{\Lambda} \mathfrak{A}$ is a projective $\Lambda^{\mathrm{op}}$-module.

(5) $P \otimes_{\Gamma} P^{*}$ is a projective $\Lambda^{\mathrm{op}}$-module.

(6) The composition $\bmod \Lambda \stackrel{(P,)}{\longrightarrow} \bmod \Gamma \stackrel{P \otimes}{\longrightarrow} \bmod \Lambda$ is exact.

The following result follows from Proposition 4.11.

Proposition 4.13. Let $\mathfrak{A}$ be an idempotent ideal and assume that p.dim ${ }_{\Lambda^{\mathrm{op}}} \mathfrak{A} \otimes_{\Lambda}$ $\mathfrak{A}=r$. Then $\mathbf{P}_{r+1}=\mathbf{P}_{r+2}=\cdots=\mathbf{P}_{\infty}$.

\section{Projectivity of $\mathfrak{A}$. Global dimension}

In this section we characterize when an idempotent ideal $\mathfrak{A}$ is a projective $\Lambda$-module, and when it is a projective $\Lambda^{\mathrm{op}}$-module. We also study relationships between the global dimensions of the algebras $\Lambda, \Lambda / \mathfrak{A}$ and $\Gamma$.

The following proposition combines results of the preceding sections.

Proposition 5.1. Let $\mathfrak{A}$ be an idempotent ideal. Then the following are equivalent

(a) $\mathfrak{A}$ is a projective $\Lambda$-module.

(b) $\mathfrak{A}$ is a strong idempotent and $\mathbf{I}_{1}=\mathbf{I}_{\infty}$.

(c) $\mathfrak{A}$ is a 2-idempotent and $\mathbf{I}_{1}=\mathbf{I}_{\infty}$.

(d) The multiplication map $\mathfrak{A} \otimes_{\Lambda} \mathfrak{A} \rightarrow \mathfrak{A}$ is an isomorphism and $\mathfrak{A} \otimes_{\Lambda} \mathfrak{A}$ is a projective $\Lambda$-module.

Proof. (a) $\Rightarrow$ (b) Assume that $\mathfrak{A}$ is a projective $\Lambda$-module. Then p.dim $\Lambda / \mathfrak{A} \leq$ 1 , so it follows from the definitions that $\mathbf{I}_{1}=\mathbf{I}_{\infty}$. And we observed in $\S 1$, Example 1 , that $\mathfrak{A}$ is a strong idempotent. So $(a) \Rightarrow(b)$. Clearly (b) $\Rightarrow(c)$. We know by Proposition 1.4 that $\mathfrak{A}$ is a strong idempotent if and only if the multiplication map $\mathfrak{A} \otimes \mathfrak{A} \rightarrow \mathfrak{A}$ is an isomorphism. On the other hand, we proved in Proposition 4.5 that $\mathbf{I}_{1}=\mathbf{I}_{\infty}$ if and only if $\mathfrak{A} \otimes_{\Lambda} \mathfrak{A}$ is projective in $\bmod \Lambda$. Then $(b) \Leftrightarrow(d)$.

We have the following characterization of when $\mathfrak{A}$ is a projective $\Lambda^{\text {op }}$ module. 
Proposition 5.2. Let $\mathfrak{A}$ be an idempotent ideal. Then the following are equivalent:

(a) $\mathfrak{A}$ is a projective $\Lambda^{\mathrm{op}}$-module.

(b) $\mathfrak{A}$ is a strong idempotent and $\mathbf{P}_{1}=\mathbf{P}_{\infty}$.

(c) $\mathfrak{A}$ is a 2-idempotent and $\mathbf{P}_{1}=\mathbf{P}_{\infty}$.

(d) The multiplication map $\mathfrak{A} \otimes_{\Lambda} \mathfrak{A} \rightarrow \mathfrak{A}$ is an isomorphism and $\mathfrak{A} \otimes_{\Lambda} \mathfrak{A}$ is a projective $\Lambda^{\mathrm{op}}$-module.

When $\Gamma$ is semisimple then $\mathbf{I}_{1}=\mathbf{I}_{\infty}$ and $\mathbf{P}_{1}=\mathbf{P}_{\infty}$, so we get the following result.

Corollary 5.3. Assume $\Gamma$ is semisimple. Then the following conditions are equivalent:

(a) $\mathfrak{A}$ is a projective $\Lambda$-module.

(b) $\mathfrak{A}$ is a 2-idempotent.

(c) $\mathfrak{A}$ is a projective $\Lambda^{\mathrm{op}}$-module.

The equivalence of (a) and (c) has been proven by Dlab and Ringel in [DR 1, Part II, Statement 7]. We observe that the conditions $\mathfrak{A}$ is a strong idempotent, $\mathbf{I}_{1}=\mathbf{I}_{\infty}$ and $\mathbf{P}_{1}=\mathbf{P}_{\infty}$ are independent, as shown in the following examples.

Let $\Lambda$ be as in Example 3, $\S 1$. That is, $\Lambda$ is the path algebra (over a field) of the quiver

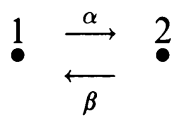

modulo the ideal generated by the relation $\beta \alpha \beta=0$. Then the ideal $\mathfrak{A}_{1}=$ $\tau_{P_{1}}(\Lambda)$ is a strong idempotent which is not projective. Therefore, $\mathbf{I}_{1}, \mathfrak{a}_{1} \neq$ $\mathbf{I}_{\infty, \mathfrak{A}_{1}}$. Let now $\mathfrak{A}_{2}=\tau_{P_{2}}(\Lambda)$ be the trace of the projective module $P_{2}$ associated to the vertex 2. Then $\mathfrak{A}_{2}$ is a projective $\Lambda$-module, so $\mathfrak{A}_{2}$ is a strong idempotent and $\mathbf{I}_{1}, \mathfrak{A}_{2}=\mathbf{I}_{\infty}, \mathfrak{a}_{2}$. However $\mathbf{P}_{1}, \mathfrak{a}_{2} \neq \mathbf{P}_{\infty}, \mathfrak{A}_{2}$. This example shows that being projective is not a symmetric condition. One can check that directly or observe that $\mathbf{P}_{1, \mathfrak{A}_{2}} \neq \mathbf{P}_{\infty, \mathfrak{A}_{2}}$ implies that $\mathfrak{A}_{2}$ is not a projective $\Lambda^{\text {op }}$-module.

On the other hand, any nonprojective idempotent ideal $\mathfrak{A}=\tau_{P} \Lambda$ such that $\Gamma$ is semisimple satisfies $\mathbf{I}_{1}=\mathbf{I}_{\infty}$ and $\mathbf{P}_{1}=\mathbf{P}_{\infty}$, but $\mathfrak{A}$ is not a 2-idempotent (Corollary 5.3). Such ideals are easy to find. One of them is the ideal $\mathfrak{A}=\tau_{P_{2}}(\Lambda)$ in $\Lambda=T_{3}(k) / r^{2}$, where $T_{3}(k)$ is the $3 \times 3$ lower triangular matrix ring with coefficients in a field $k, P_{2}=\Lambda e_{22}$ and $e_{22}$ is the matrix with 1 in the entry 22, 0 elsewhere.

We now study relationships between the global dimensions of the rings $\Lambda$, $\Lambda / \mathfrak{A}$ and $\Gamma$. We have seen that if the idempotent ideal $\mathfrak{A}$ is a strong idempotent then $\operatorname{gl} \cdot \operatorname{dim} \Lambda / \mathfrak{A} \leq \operatorname{gl} \cdot \operatorname{dim} \Lambda$. And if $\mathbf{I}_{1}=\mathbf{I}_{\infty}$ then $\operatorname{gl} \cdot \operatorname{dim} \Gamma \leq \operatorname{gl} \cdot \operatorname{dim} \Lambda$.

We will prove now the following result.

Theorem 5.4. Let $\mathfrak{A}$ be an idempotent ideal. Then $\operatorname{gl} \cdot \operatorname{dim} \Lambda \leq p \cdot \operatorname{dim}_{\Lambda} \Lambda / \mathfrak{A}+$ gl.dim $\Lambda / \mathfrak{A}+\operatorname{gl} . \operatorname{dim} \Gamma+1$.

We observe that when $\mathfrak{A}$ is projective and $\Gamma$ is semisimple the proposition states that $\operatorname{gl} . \operatorname{dim} \Lambda \leq \operatorname{gl} . \operatorname{dim} \Lambda / \mathfrak{A}+2$, an inequality proven by Dlab and Ringel in [DR1, Part 2, Statement 5].

To prove this proposition and the next we consider the full subcategory $T$ of $\bmod \Lambda$ consisting of the modules $T$ such that $\operatorname{Ext}_{\Lambda}^{i}(\Lambda / \mathfrak{A}, T)=0$ for all 
$i \geq 1$. Then $\mathbf{I}_{\infty} \subseteq \mathbf{T}$, and a $\Lambda$-module $T$ in $\mathbf{I}_{\infty}$ is in $\mathbf{T}$ if and only if $\tau_{\Lambda / \mathfrak{A}} T=$ $\operatorname{Hom}_{\Lambda}(\Lambda / \mathfrak{A}, T)=0$. Given a $\Lambda$-module $X$ we denote by $\Omega^{-i}(X)$ the image of the map $d_{i}$ in a minimal injective coresolution $0 \rightarrow X \rightarrow I_{0} \stackrel{d_{1}}{\longrightarrow} I_{1} \stackrel{d_{2}}{\longrightarrow} \ldots$ of $X$ and we write $\Omega^{0}(X)=X$. We need the following lemma.

Lemma 5.5. Let $\mathrm{T}$ be as above. Then

(i) $\mathbf{p} \cdot \operatorname{dim}_{\Lambda} \Lambda / \mathfrak{A}=r$ if and only if $\Omega^{-r}(\bmod \Lambda) \subseteq \mathbf{T}$.

(ii) If $\operatorname{gl} \cdot \operatorname{dim} \Lambda / \mathfrak{A}=s<\infty$ then $\Omega^{-(s+1)}(\mathbf{T}) \subseteq \mathbf{I}_{\infty}$.

Proof. (i) Follows from the isomorphism

$$
\operatorname{Ext}_{\Lambda}^{i+r}(\Lambda / \mathfrak{A}, X) \simeq \operatorname{Ext}_{\Lambda}^{i}\left(\Lambda / \mathfrak{A}, \Omega^{-r} X\right),
$$

for all $X$ in $\bmod \Lambda, i \geq 1$.

(ii) Let $T$ in $\mathbf{T}$. Clearly $\Omega^{-i}(T)$ is in $\mathbf{T}$ for all $i \geq 1$. So we only have to prove that $\tau_{\Lambda / \mathfrak{A}}\left(\Omega^{-(s+1)}(T)\right)=0$. Let $0 \rightarrow T \rightarrow I_{0} \rightarrow I_{1} \rightarrow \cdots$ be a minimal injective coresolution of $T$. Since $T$ is in $T$ we have $0 \rightarrow$ $\tau_{\Delta / \mathfrak{A}} T \rightarrow \tau_{\Lambda / \mathfrak{A}} I_{0} \rightarrow \tau_{\Delta / \mathfrak{A}} I_{1} \rightarrow \cdots$ is a minimal injective coresolution of $\tau_{\Lambda / \mathfrak{A}} T$ (Proposition 1.1). Since gl.dim $\Lambda / \mathfrak{A}=s$ it follows that $\tau_{\Lambda / \mathfrak{A}} I_{k}=0$ for $k>s$. So $\tau_{\Lambda / \mathfrak{A}}\left(\Omega^{-(s+1)}(T)\right) \subseteq \tau_{\Lambda / \mathfrak{A}}\left(I_{s+1}\right)=0$, as we want to prove.

We prove now the theorem. Let $r=p \cdot \operatorname{dim}_{\Lambda} \Lambda / \mathfrak{A}, s=\operatorname{gl} \operatorname{dim} \Lambda / \mathfrak{A}, t=$ gl.dim $\Gamma$. Let $X$ be in $\bmod \Lambda$. Using first (i) and then (ii) of Lemma 5.5, we conclude that $\Omega^{-(r+s+1)}(X)$ is in $\mathbf{I}_{\infty}$. Then

$$
\text { inj.dim } \Omega^{-(r+s+1)}(X)=\text { inj. } \operatorname{dim}_{\Gamma}\left(P, \Omega^{-(r+s+1)}(X)\right),
$$

by Corollary 3.3. This number is at most $t=\operatorname{gl} \cdot \operatorname{dim} \Gamma$. So inj.dim $X \leq r+s+$ $t+1$, and the proof of the theorem is complete.

When $\mathfrak{A}$ is a projective $\Lambda$-module we obtain the following result

Corollary 5.6. Assume the idempotent ideal $\mathfrak{A}$ is a projective $\Lambda$-module. Then the following are equivalent

(a) $\operatorname{gl} \operatorname{dim} \Lambda<\infty$,

(b) $\operatorname{gl} \cdot \operatorname{dim} \Lambda / \mathfrak{A}<\infty$ and $\operatorname{gl} \cdot \operatorname{dim} \Gamma<\infty$.

Proof. (a) $\Rightarrow$ (b) follows from the fact that $\mathfrak{A}$ is projective if and only if $\mathfrak{A}$ is a strong idempotent and $\mathbf{I}_{1}=\mathbf{I}_{\infty}$, proven in Proposition 5.1. (b) $\Rightarrow(\mathrm{a})$ is an immediate consequence of the proposition.

When the ideal $\mathfrak{A}$ is a strong idempotent we can prove a stronger inequality.

Proposition 5.7. Let $\mathfrak{A}$ be a strong idempotent ideal. Then

gl.dim $\Lambda \leq p \cdot \operatorname{dim}_{\Lambda} \Lambda / \mathfrak{A}+\max \left\{p \cdot \operatorname{dim}_{\Lambda_{\text {op }} \Lambda / \mathfrak{A}}+\operatorname{gl} \cdot \operatorname{dim} \Lambda / \mathfrak{A}, \operatorname{gl} \cdot \operatorname{dim} \Gamma\right\}$.

Proof. We start by stating the following lemma.

Lemma 5.8. Let $\mathfrak{A}$ be a two-sided ideal and let $X$ be a $\Lambda / \mathfrak{A}$-module. Then

(i) $p \cdot \operatorname{dim}_{\Lambda} X \leq p \cdot \operatorname{dim}_{\Lambda / \mathfrak{A}} X+p \cdot \operatorname{dim}_{\Lambda} \Lambda / \mathfrak{A}$,

(ii) inj.dim $\operatorname{din}_{\Lambda} X$ inj.dim $\operatorname{din}_{\Lambda / \mathfrak{A}} X+\mathrm{p} \cdot \operatorname{dim}_{\Lambda^{\mathrm{op}}} \Lambda / \mathfrak{A}$.

Proof. (i) follows by induction on $n=\mathfrak{p} \cdot \operatorname{dim}_{\Lambda / \mathfrak{A}} X$, and (ii) follows from (i) by duality.

Now we prove the proposition. We assume that $\mathfrak{A}$ is a strong idempotent ideal, and that $X$ is a $\Lambda$-module. We may assume that $r=\operatorname{p}^{-\operatorname{dim}_{\Lambda} \Lambda / \mathfrak{A}}<\infty$. Then $T=\Omega^{-r} X$ is in $T$. We consider the exact sequence $0 \rightarrow \tau_{\Lambda / \mathfrak{A}} T \rightarrow$ 
$T \rightarrow T / \tau_{\Lambda / \mathfrak{A}} T \rightarrow 0$. Since $T$ is in $\mathbf{T}, \tau_{\Lambda / \mathfrak{A}} T$ is a $\Lambda / \mathfrak{A}$-module and $\mathfrak{A}$ is a strong idempotent, it follows that $T / \tau_{\Lambda / \mathfrak{A}} T$ is in $\mathbf{I}_{\infty}$. Thus inj.dim $T / \tau_{\Lambda / \mathfrak{A}} T \leq$ gl.dim $\Gamma$. On the other hand we get by (ii) of Lemma 5.8 that inj.dim $\operatorname{dim}_{\Lambda}\left(\tau_{\Lambda / \mathfrak{A}} T\right)$ $\leq \mathrm{gl} \cdot \operatorname{dim} \Lambda / \mathfrak{A}+\mathrm{p} \cdot \operatorname{dim}_{\Lambda^{\mathrm{op}}} \Lambda / \mathfrak{A}$. Combining these results we get

$$
\text { inj.dim } \operatorname{dim}_{\Lambda} T \leq \max \left\{\operatorname{gl} \cdot \operatorname{dim} \Lambda / \mathfrak{A}+\text { p.dim } \Lambda_{\Lambda_{\mathrm{op}}} \Lambda / \mathfrak{A}, \operatorname{gl} \cdot \operatorname{dim} \Gamma\right\} .
$$

This inequality proves the proposition because $T=\Omega^{-r} X$, so inj.dim $X=$ $r+\operatorname{inj} . \operatorname{dim} T$.

We observe now that if $\mathfrak{A}$ is a strong idempotent then $\mathfrak{A}$ is in $\mathbf{P}_{\infty}$, so p.dim $\operatorname{dim}_{\Lambda} \mathfrak{A} \operatorname{p\cdot dim} \Gamma(P, \mathfrak{A}) \leq \operatorname{gl} \cdot \operatorname{dim} \Gamma$. Since being a strong idempotent is a symmetric condition we also have that $p . \operatorname{dim}_{\Lambda_{\text {op }}} \mathfrak{A} \leq \operatorname{gl} \cdot \operatorname{dim} \Gamma$. Combining the inequality in Proposition 5.7 with $\mathrm{p} \cdot \operatorname{dim}_{\Lambda^{\mathrm{op}}} \Lambda / \mathfrak{A} \leq \operatorname{gl} \cdot \operatorname{dim} \Gamma+1$, we obtain the inequality of Theorem 5.4 .

As an immediate consequence of Proposition 5.7 we obtain the following corollary.

Corollary 5.9. Assume that the idempotent ideal $\mathfrak{A}$ is projective in $\bmod \Lambda$ and in $\bmod \Lambda^{\mathrm{op}}$. Then

$$
\operatorname{gl} \cdot \operatorname{dim} \Lambda \leq \max \{\operatorname{gl} \cdot \operatorname{dim} \Lambda / \mathfrak{A}+2, \operatorname{gl} \cdot \operatorname{dim} \Gamma+1\} .
$$

\section{QUASIHEREDITARY ALGEBRAS}

In this section we apply some of our previous results to quasihereditary algebras. First we show that being quasihereditary is invariant under Morita equivalence. Second, we study the projective resolutions of the ideals in a heredity chain. We give another proof of the fact that hereditary artin algebras can be characterized as quasihereditary artin algebras for which each chain of idempotent ideals can be refined to a heredity chain, which was shown by Dlab and Ringel in [DR1]. Finally we give a characterization of quasihereditary algebras using $l$-hereditary algebras.

We recall now the definition of quasihereditary algebras [CPS]: An artin algebra $\Lambda$ is quasihereditary if there is a chain of idempotent ideals of $\Lambda$, $0=\mathfrak{A}_{0} \subset \cdots \subset \mathfrak{A}_{n}=\Lambda$ satisfying the following conditions

(QH1) $\mathfrak{A}_{i} / \mathfrak{A}_{i-1}$ is projective in $\bmod \left(\Lambda / \mathfrak{A}_{i-1}\right)$ for all $i=1, \ldots, n$.

$(\mathrm{QH} 2) \Gamma_{i}=\operatorname{End}_{\Lambda / \mathfrak{A}_{i-1}}\left(Q_{i} / \mathfrak{A}_{i-1} Q_{i}\right)^{\text {op }}$ is semisimple for all $i=1, \ldots, n$, where $Q_{i}$ is a projective $\Lambda$-module such that $\mathfrak{A}_{i}=\tau_{Q_{i}}(\Lambda)$.

Such chain is called an heredity chain.

Let $\mathfrak{A}=\tau_{P} \Lambda$ be an idempotent ideal of $\Lambda$. We proved in Corollary 5.3 that if $\Gamma=\operatorname{End}(P)^{\text {op }}$ is a semisimple ring, then $\mathfrak{A}$ is projective in $\bmod \Lambda$ if and only if $\mathfrak{A}$ is 2-idempotent. So the conditions (QH1) in the definition of quasihereditary algebra can be replaced by either of the two following conditions

$\left(\mathrm{QH} 1^{\prime}\right) \mathfrak{A}_{i}$ is 2-idempotent, for all $i=1, \ldots, n$.

$\left(\mathrm{QH} 1^{\prime \prime}\right) \mathfrak{A}_{i}$ is strong idempotent, for all $i=1, \ldots, n$.

We use this observation to prove that being quasihereditary is a Morita invariance, result which was proven in [CPS] using highest weight categories. As we noticed in the introduction giving an idempotent ideal $\mathfrak{A}$ of $\Lambda$ is equivalent to giving a Serre subcategory of $\bmod \Lambda$. When $\mathfrak{A}=\tau_{P}(\Lambda)$ is an 
idempotent ideal of $\Lambda$ then $S=\bmod \Lambda / \mathfrak{A}$ is a Serre subcategory of $\bmod \Lambda$ and $\bmod \Lambda / \mathfrak{A} \rightarrow \bmod \Lambda \rightarrow \bmod \Gamma$ is an exact sequence of categories, where $\Gamma=\operatorname{End}(P)^{\text {op }}$.

Since the properties of being strong idempotent and of being semisimple are Morita invariances it follows that being quasihereditary is also a Morita invariance.

In the following proposition we give a characterization of the chains $0=$ $\mathfrak{A}_{0} \subset \mathfrak{A}_{1} \subset \cdots \subset \mathfrak{A}_{n}$ of idempotent ideals of $\Lambda$ such that $\mathfrak{A}_{i}$ is projective for $i=1, \ldots, n$, in terms of the projective presentations of the ideals $\mathfrak{A}_{i}$ in the chain.

Proposition 6.1. Let $0=\mathfrak{A}_{0} \subset \mathfrak{A}_{1} \subset \cdots \subset \mathfrak{A}_{n}$ be a chain of idempotent ideals in $\Lambda$. Then there exist projective $\Lambda$-modules $P_{1}, \ldots, P_{n}$ such that $\mathfrak{A}_{k}=$ $\tau_{P_{1} \amalg \cdots \amalg P_{k}}(\Lambda)$ for all $k=1, \ldots, n$. Moreover, the following conditions are equivalent:

(a) $\mathfrak{A}_{k} / \mathfrak{A}_{k-1}$ is projective for all $k=1, \ldots, n$.

(b) $\mathfrak{A}_{k}$ has a projective resolution $\cdots \rightarrow P_{k, i} \rightarrow \cdots \rightarrow P_{k, 0} \rightarrow \mathfrak{A}_{k} \rightarrow 0$, with $P_{k, i}$ in $\operatorname{add}\left(P_{1} \amalg \cdots \amalg P_{k-i}\right)$ for all $i<k, P_{k, i}=0$ for $i \geq k$, for all $k=1, \ldots, n$.

(c) $\mathfrak{A}_{k}$ has a projective presentation $P_{k, 1} \rightarrow P_{k, 0} \rightarrow \mathfrak{A}_{k} \rightarrow 0$, with $P_{k, 1}$ in $\operatorname{add}\left(P_{1} \amalg \cdots \amalg P_{k-1}\right)$, for all $k=1, \ldots, n$.

Proof. The first statement follows from the following observation. If $P, Q$ are projective $\Lambda$-modules and $\tau_{P}(\Lambda) \subset \tau_{Q}(\Lambda)$, then $P$ is in add $Q$.

To prove the equivalence of $(a)$ and $(b)$ we introduce the following notation. For $k \geq 1$, let $\mathbf{R}_{k}$ be the full subcategory of $\bmod \Lambda$ consisting of the $\Lambda$ modules $X$ having a projective resolution $\cdots \rightarrow Q_{i} \rightarrow \cdots \rightarrow Q_{0} \rightarrow X \rightarrow 0$, with $Q_{i}$ in $\operatorname{add}\left(P_{1} \amalg \cdots \amalg P_{k-i}\right)$ if $i \leq k-1, Q_{i}=0$ otherwise. We observe that $\mathbf{R}_{k}$ is closed under extensions.

We prove that $(\mathbf{a}) \Rightarrow(\mathrm{b})$ by induction on $n$ It is clearly true for $n=1$. So let $n>1$ and consider the sequence $0 \rightarrow \mathfrak{A}_{n-1} \rightarrow \mathfrak{A}_{n} \rightarrow \mathfrak{A}_{n} / \mathfrak{A}_{n-1} \rightarrow 0$. To prove (b) we have to prove that $\mathfrak{A}_{n}$ is in $\mathbf{R}_{n}$. By the induction hypothesis we know that $\mathfrak{A}_{n-1}$ is in $\mathbf{R}_{n-1} \subset \mathbf{R}_{n}$. Since $\mathbf{R}_{n}$ is closed under extensions it is enough to see that $\mathfrak{A}_{n} / \mathfrak{A}_{n-1}$ is in $\mathbf{R}_{n}$. We know by (a) that $\mathfrak{A}_{n} / \mathfrak{A}_{n-1}$ is a projective $A / \mathfrak{A}_{n-1}$-module. So $\mathfrak{A}_{n} / \mathfrak{A}_{n-1} \simeq Q_{n} / \mathfrak{A}_{n-1} Q_{n}$, where $Q_{n}$ is the projective cover of $\mathfrak{A}_{n} / \mathfrak{A}_{n-1}$ in $\bmod \Lambda$. Since $Q_{n}$ is in add $\Lambda$, then $\mathfrak{A}_{n-1} Q_{n}$ is in add $\mathfrak{A}_{n-1} \subseteq$ $\mathbf{R}_{n}$. Thus from the exact sequence $0 \rightarrow \mathfrak{A}_{n-1} Q_{n} \rightarrow Q_{n} \rightarrow \mathfrak{A}_{n} / \mathfrak{A}_{n-1} \rightarrow 0$ with $Q_{n}$ in add $P_{0}\left(\mathfrak{A}_{n}\right)=\operatorname{add}\left(P_{1} \amalg \cdots \amalg P_{n}\right)$ we get that $\mathfrak{A}_{n} / \mathfrak{A}_{n-1}$ is in $\mathbf{R}_{n}$. This finishes the proof of $(a) \Rightarrow(b)$.

(b) $\Rightarrow$ (c) is clear. So we prove now that (c) $\Rightarrow\left(\right.$ a). Let $P_{k, 1} \rightarrow P_{k, 0} \rightarrow \mathfrak{A}_{k} \rightarrow 0$ be a presentation of $\mathfrak{A}_{k}$ with $P_{k, 1}$ in $\operatorname{add}\left(P_{1} \amalg \cdots \amalg P_{k-1}\right), 1 \leq k \leq n$. We consider the exact sequence $0 \rightarrow K_{k} \rightarrow P_{k, 0} \rightarrow \mathfrak{A}_{k} \rightarrow 0$. Tensoring with $\Lambda / \mathfrak{A}_{k-1}$ we get an exact sequence

$$
K_{k} / \mathfrak{A}_{k-1} K_{k} \rightarrow P_{0, k} / \mathfrak{A}_{k-1} P_{k, 0} \stackrel{\bar{\varphi}_{k}}{\longrightarrow} \mathfrak{A}_{k} / \mathfrak{A}_{k-1} \rightarrow 0 .
$$

Then $P_{0}\left(K_{k}\right) \simeq P_{k, 1}$ is in $\operatorname{add}\left(P_{1}, \ldots, P_{k-1}\right)$, by (c). That is, $\mathfrak{A}_{k-1} K_{k}=K_{k}$. So $\bar{\varphi}_{k}$ is an isomorphism and $\mathfrak{A}_{k} / \mathfrak{A}_{k-1}$ is projective in $\bmod \left(\Lambda / \mathfrak{A}_{k-1}\right)$.

The following corollary applies to heredity chains in a quasihereditary algebra. 
Corollary 6.2. Let $0=\mathfrak{A}_{0} \subset \cdots \subset \mathfrak{A}_{n}$ be a chain of idempotent ideals in $\Lambda$ such that $\mathfrak{A}_{k} / \mathfrak{A}_{k-1}$ is a projective $\Lambda / \mathfrak{A}_{k-1}$ module for all $k=1, \ldots, n$. Then p.dim $\mathfrak{A}_{k} \leq k-1, \mathfrak{A}_{k}$ is strong idempotent, and $\mathbf{I}_{k, \mathfrak{x}_{k}}=\mathbf{I}_{\infty, \mathfrak{A}_{k}}$, for $k=$ $1, \ldots, n$.

Proof. The equivalence of (a) and (b) in Proposition 6.1 implies that $p . \operatorname{dim}_{\Lambda} \mathfrak{A}_{k}$ $\leq k-1$ and that $\mathfrak{A}_{k}$ has a projective resolution in $\operatorname{add}\left(P_{0}\left(\mathfrak{A}_{k}\right)\right)$. By Theorem 2.1 we know that this implies that $\mathfrak{A}_{k}$ is a strong idempotent. Then $\mathfrak{A}_{k}$ is a 2idempotent, so $\mathfrak{A}_{k} \otimes_{\Lambda} \mathfrak{A}_{k} \simeq \mathfrak{A}_{k}$, as proven in Proposition 1.4. Then p.dim $\mathfrak{A}_{k} \otimes_{\Lambda}$ $\mathfrak{A}_{k} \leq k$, so that $\mathbf{I}_{k, \mathfrak{A}_{k}}=\mathbf{I}_{\infty, \mathfrak{A}_{k}}$ (Proposition 4.11).

As a consequence of the above proposition we prove the following result of Dlab and Ringel.

Proposition 6.3 [DR1, Part 1, Theorem 1]. An artin algebra $\Lambda$ is hereditary if and only if any chain of idempotent ideals in $\Lambda$ can be refined to a heredity chain.

Proof. Assume that any chain of idempotent ideals can be refined to a heredity chain. We will prove that $\Lambda$ is hereditary.

Let $Q$ be an indecomposable projective $\Lambda$-module. To prove that $\Lambda$ is hereditary we prove that $r Q$ is projective. Let $P$ be the sum of all the indecomposable nonisomorphic projective modules not isomorphic to $Q$. Then $\mathfrak{A}=\tau_{P}(\Lambda)$ is a maximal proper idempotent ideal of $\Lambda$, so by hypothesis it is part of a heredity chain. By the maximality of $\mathfrak{A}$ it follows that it must be the last proper ideal in the chain, so that the ring $\Lambda / \mathfrak{A}$ is semisimple. Then $Q / \mathfrak{A} Q$ is a simple $\Lambda$-module, so $r Q=\mathfrak{A} Q$.

The module $\mathfrak{A} Q$ is in add $\mathfrak{A}$ because $Q$ is in add $\Lambda$. Therefore to prove that $r Q=\mathfrak{A} Q$ is projective it is enough to prove that $\mathfrak{A}$ is projective. Let $P_{1} \rightarrow P_{0} \rightarrow \mathfrak{A} \rightarrow 0$ be a minimal projective presentation of $\mathfrak{A}$ in $\bmod \Lambda$. We will prove that $P_{1}=0$. Let $Q_{1}$ be an indecomposable direct summand of $P$. We write $P=Q_{1} \amalg P^{\prime}$. The chain of idempotent ideals $\tau_{P^{\prime}}(\Lambda) \subset \tau_{P}(\Lambda)$ can be refined to a heredity chain $0=\mathfrak{A}_{0} \subset \cdots \subset \mathfrak{A}_{n}=\Lambda$. Let $k$ be such that $\mathfrak{A}_{k}=\tau_{P}(\Lambda)=\mathfrak{A}$. Since $Q_{1}$ is indecomposable we must have $\mathfrak{A}_{k-1}=\tau_{P^{\prime}}(\Lambda)$. We can apply now Proposition 6.1 to the chain $\mathfrak{A}_{0} \subset \cdots \subset \mathfrak{A}_{k}=\mathfrak{A}$ and the given projective presentation $P_{1} \rightarrow P_{0} \rightarrow \mathfrak{A} \rightarrow 0$ of $\mathfrak{A}$. We conclude that $P_{1}$ is in add $P^{\prime} \subseteq$ add $P$ and therefore has no summand isomorphic to $Q_{1}$. Therefore we have proven that no indecomposable summand $Q_{1}$ of $P$ is in add $P_{1} \subseteq$ add $P$. So $P_{1}=0$. This finishes the proof that $\Lambda$ is hereditary.

So we have proven that if any chain of idempotent ideals can be refined to a heredity chain then $\Lambda$ is hereditary. The converse follows from the fact that any chain of idempotent ideals can be refined to a chain $0 \subset \tau_{P_{1}} \subset \cdots \subset$ $\tau_{P_{1} \amalg \cdots \amalg P_{n}} \subset \Lambda$, where all the projective $\Lambda$-modules $P_{i}$ are indecomposable.

We know by [BF, Proposition 1.6], that $l$-hereditary algebras are quasihereditary. We give now a characterization of quasihereditary algebras using $l$-hereditary algebras.

We recall that an artin algebra $\Lambda$ is $l$-hereditary if the maps between indecomposable projective modules are either zero or monomorphisms. If $\Lambda$ is an $l$-hereditary algebra and $P$ is a projective $\Lambda$-module, then the algebra $\Gamma=\operatorname{End}_{\Lambda}(P)^{\text {op }}$ is also $l$-hereditary. 
Proposition 6.4. Let $\Lambda$ be an artin algebra with a chain of idempotent ideals $0=\mathfrak{A}_{0} \subset \mathfrak{A}_{1} \subset \cdots \subset \mathfrak{A}_{n}=\Lambda$ such that (1) $\mathfrak{A}_{i} / \mathfrak{A}_{i-1}$ is a projective $\Lambda / \mathfrak{A}_{i-1}$ module for $i=1, \ldots, n$ and (2) $\operatorname{End}_{\Lambda / \mathfrak{A}_{i-1}}\left(Q_{i} / \mathfrak{A}_{i-1} Q_{i}\right)$ is l-hereditary for $i=1, \ldots, n$, where $Q_{i}$ is a projective $\Lambda$-module such that $\tau_{Q_{i}}(\Lambda)=\mathfrak{A}_{i}$. Then $\Lambda$ is quasihereditary.

Proof. The proposition follows by induction from the following lemma.

Lemma 6.5. Let $P$ be a projective $\Lambda$-module such that $\mathfrak{A}=\tau_{P}(\Lambda)$ is a projective $\Lambda$-module and $\Gamma=\operatorname{End}_{\Lambda}(P)^{\text {op }}$ is an l-hereditary algebra. If $\Gamma$ is not semisimple, then there exists a direct summand $P_{1}$ of $P$ such that $\mathfrak{A}_{1}=\tau_{P_{1}}(\Lambda)$ is a projective $\Lambda$-module, $\operatorname{End}_{\Lambda}\left(P_{1}\right)$ is semisimple and $\operatorname{End}_{\Lambda / \mathfrak{A}_{1}}\left(P / \mathfrak{A}_{1} P\right)$ is an l-hereditary algebra.

Proof. Let $P_{1}$ be an indecomposable summand of $P$ such that the $\Gamma$-module $\left(P, P_{1}\right)$ has maximum length. Let $P_{1}, \ldots, P_{n}$ be the nonisomorphic indecomposable summands of $P$. Since $\Gamma$ is $l$-hereditary it follows that

$$
\left(\left(P, P_{1}\right),\left(P, P_{i}\right)\right)=0 \text { for } i=2, \ldots, r .
$$

We will see that $P_{1}$ has the desirec properties.

Since

$$
\operatorname{End}_{\Lambda}\left(P_{1}\right) \simeq \operatorname{End}_{\Gamma}\left(\left(P, P_{1}\right)\right)
$$

and $\Gamma$ is $l$-hereditary it follows that $\operatorname{End}_{\Lambda}\left(P_{1}\right)$ is a division ring. On the other hand, $\operatorname{Hom}_{\Lambda}\left(P_{1}, P_{i}\right)=\operatorname{Hom}_{\Gamma}\left(\left(P, P_{1}\right),\left(P, P_{i}\right)\right)=0$ for $i=2, \ldots, n$, so $\tau_{P_{1}}\left(P_{i}\right)=0$ if $i \geq 2$. Thus $\tau_{P_{1}}(X)$ is in add $P_{1}$, for any $X$ in add $P$. Now, $\tau_{P_{1}}(\Lambda)=\tau_{P_{1}}\left(\tau_{P}(\bar{\Lambda})\right)$, and $\tau_{P}(\Lambda)$ is in add $P$ since it is projective by hypothesis. So $\tau_{P_{1}}(\Lambda)$ is in add $P_{1}$ and is thus projective.

Since $\mathfrak{A}_{1} P=\tau_{P_{1}}(P)$ is in add $P_{1}$ it follows that $P / \mathfrak{A}_{1} P$ is in

$$
\operatorname{add}\left(P_{1} \amalg \cdots \amalg P_{n}\right) \subseteq \text { add } P .
$$

Therefore $\operatorname{End}_{\Lambda}\left(P / \mathfrak{A}_{1} P\right)=\operatorname{End}_{\Gamma}\left(\left(P, P / \mathfrak{A}_{1} P\right)\right)$ and $\left(P, P / \mathfrak{A}_{1} P\right)$ is in add $\Gamma$. Since $\Gamma$ is $l$-hereditary it follows then that $\operatorname{End}_{\Lambda}\left(P / \mathfrak{A}_{1} P\right)$ is $l$-hereditary. This ends the proof of the lemma.

Using this characterization of quasihereditary algebras we obtain a bound for the global dimension of a quasihereditary algebra, different from the one given in [DR1, Statement 9].

Corollary 6.6. Let $\Lambda$ be a quasihereditary algebra. Let $0=\mathfrak{A}_{0} \subset \cdots \subset \mathfrak{A}_{n}=\Lambda$ with $\mathfrak{A}_{i}=\tau_{P_{i}}(\Lambda)$ be a chain of idempotent ideals such that $\mathfrak{A}_{i} / \mathfrak{A}_{i-1}$ is projective in $\bmod \left(\Lambda / \mathfrak{A}_{i-1}\right)$ and $\operatorname{End}_{\Lambda / \mathfrak{A}_{i}}\left(P_{i} / \mathfrak{A}_{i-1} P_{i}\right)$ is hereditary for $i=1, \ldots, n$. Then gl.dim $\Lambda \leq 3 n-2$.

Proof. We prove the corollary by induction on $n$. If $n=1$ then $\Lambda$ is hereditary and the formula holds. Let $n>1$. We apply Theorem 5.4 to the idempotent ideal $\mathfrak{A}=\mathfrak{A}_{1}=\tau_{P_{1}}(\Lambda)$ and we obtain that

$$
\operatorname{gl} \cdot \operatorname{dim} \Lambda \leq \mathrm{p} \cdot \operatorname{dim} \Lambda \Lambda / \mathfrak{A}_{1}+\operatorname{gl} \cdot \operatorname{dim} \Lambda / \mathfrak{A}_{1}+\operatorname{gl} \cdot \operatorname{dim} \Gamma+1,
$$

where $\Gamma=\operatorname{End}_{\Lambda}\left(P_{1}\right)$ is hereditary by hypothesis. The induction hypothesis applied to the chain $0 \subset \mathfrak{A}_{2} / \mathfrak{A}_{1} \subset \cdots \subset \mathfrak{A}_{n} / \mathfrak{A}_{1}=\Lambda / \mathfrak{A}_{1}$ in $\bmod \left(\Lambda / \mathfrak{A}_{1}\right)$ implies that $\operatorname{gl} \cdot \operatorname{dim} \Lambda / \mathfrak{A}_{1} \leq 3(n-1)-2$. Thus $\operatorname{gl} \cdot \operatorname{dim} \Lambda \leq 3(n-1)-2+3=3 n-2$. 


\section{A CLASS OF QUASIHEREDITARY ALGEBRAS}

We define in this section a class of artin algebras which contains several well-known classes of algebras and prove that the algebras in that class are quasihereditary.

Definition 7.1. We say that an artin algebra $\Lambda$ satisfies the descending Loewy length condition on projective resolutions if for every $\Lambda$-module $M$ a minimal projective resolution $\rightarrow P_{i}(M) \rightarrow \cdots \rightarrow P_{0}(M) \rightarrow M \rightarrow 0$ satisfies $L L\left(P_{i+1}(M)\right)<L L\left(P_{i}(M)\right)$ for all $i \geq 1$. Here $L L$ denotes the Loewy length.

The following are examples of classes of algebras satisfying the descending Loewy length condition on projective resolutions:

(1) $l$-hereditary algebras, which were defined in the previous section.

(2) Artin algebras of global dimension two.

(3) The endomorphism algebra $\Gamma=\operatorname{End}_{\Lambda}\left(\Lambda / r \amalg \cdots \amalg \Lambda / r^{n}\right)$, where $\Lambda$ is an artin algebra and $n$ is the index of nilpotency of the radical $r$ of $\Lambda$ [A, Proof of Proposition 10.2].

By proving that algebras satisfying the descending Loewy length condition on projective resolutions are quasihereditary, we give, in particular, a unified proof of the results, proven by V. Dlab and C. M. Ringel in [DR1, DR2], that the algebras of global dimension 2 and the endomorphism algebra $\Gamma$ of example (3) are quasihereditary.

We start by proving the following lemma.

Lemma 7.2. Let $\Lambda$ be an artin algebra satisfying the descending Loewy length condition on projective resolutions. Let $X$ be a submodule of a projective $\Lambda$ module. Then $L L\left(P_{1}(X)\right)<L L\left(P_{0}(X)\right)$.

Proof. Follows easily from the definitions.

Theorem 7.3. Let $\Lambda$ be an artin algebra satisfying the descending Loewy length condition on projective resolutions. Then $\Lambda$ is quasihereditary.

Proof. To prove this theorem we will use the characterization of quasihereditary algebras given in Proposition 6.4 which states: $\Lambda$ is quasihereditary if and only if there is a chain of idempotent ideals $0=\mathfrak{A}_{0} \subset \mathfrak{A}_{1} \subset \cdots \subset \mathfrak{A}_{n}=\Lambda$ such that (1) $\mathfrak{A}_{i} / \mathfrak{A}_{i-1}$ is a projective $\Lambda / \mathfrak{A}_{i-1}$-module for $i=1, \ldots, n$ and (2) $\operatorname{End}_{\Lambda / \mathfrak{A}_{n-1}}\left(Q_{i} / \mathfrak{A}_{i-1} Q_{i}\right)$ is $l$-hereditary for $i=1, \ldots, n$. Here $\mathfrak{A}_{i}=\tau_{Q_{i}}(\Lambda)$, and all $Q_{i}$ are projective $\Lambda$-modules.

Let $i_{1}<\cdots<i_{t}$ be the Loewy lengths of the indecomposable projective $\Lambda$ modules. Let $Q_{k}$ be the sum of the nonisomorphic indecomposable projective $\Lambda$-modules of Loewy length at most $i_{k}$, and $\mathfrak{A}_{k}=\tau_{Q_{k}}(\Lambda)$ for $k=1, \ldots, t$, $\mathfrak{A}_{0}=0$. Since $\mathfrak{A}_{k}$ is a submodule of $\Lambda$ it follows from Lemma 7.2 that $L L\left(P_{1}\left(\mathfrak{A}_{k}\right)\right)<L L\left(P_{0}\left(\mathfrak{A}_{k}\right)\right) \leq i_{k}$. So $P_{1}\left(\mathfrak{A}_{k}\right)$ is in add $Q_{k-1}$. This proves that $\mathfrak{A}_{k} / \mathfrak{A}_{k-1}$ is projective in $\bmod \left(\Lambda / \mathfrak{A}_{k-1}\right)$.

We will show in the following lemma that $\operatorname{End}_{\Lambda / \mathfrak{x}_{k-1}}\left(Q_{k} / \mathfrak{A}_{k-1} Q_{k}\right)$ is $l$ hereditary for all $k$. This will end the proof of the theorem.

Lemma 7.4. Let $\Lambda$ be an artin algebra satisfying the descending Loewy length condition on projective resolutions. Let $r$ be the Loewy length of an indecomposable projective $\Lambda$-module. Let $Q$ be the sum of the indecomposable nonisomorphic projective $\Lambda$-modules of Loewy length $r$. Let $P$ be the sum of the 
indecomposable nonisomorphic projective $\Lambda$-modules of Loewy length smaller than $r$, and $\mathfrak{A}=\tau_{P}(\Lambda)$. Then $\operatorname{End}_{\Lambda / \mathfrak{A}}(Q / \mathfrak{A} Q)$ is an l-hereditary algebra.

Proof. Let $\bar{f}: Q_{1} / \mathfrak{A} Q_{1} \rightarrow Q_{2} / \mathfrak{A} Q_{2}$ be a $\Lambda / \mathfrak{A}$-morphism. Then $\bar{f}$ is induced by a $\Lambda$-morphism $f: Q_{1} \rightarrow Q_{2}$. Let $Q_{1} \stackrel{p}{\longrightarrow} \operatorname{Im} f \stackrel{j}{\longrightarrow} Q_{2}$ be the factorization of $f$ through the image of $f$. Then $\bar{f}=\bar{j} \cdot \bar{p}$ where $p: Q_{1} / \mathfrak{A} Q_{1} \rightarrow \operatorname{Im} f / \mathfrak{A} \operatorname{Im} f$ and $\bar{j}: \operatorname{Im} f / \mathfrak{A} \operatorname{Im} f \rightarrow Q_{2} / \mathfrak{A} Q_{2}$ are the maps induced by $p$ and $j$. We will show first that if $Q_{1}$ is in add $Q$ then $\bar{p}$ is an isomorphism. And we will show that if $Q_{2}$ is in add $Q$ then $\bar{j}$ is either zero or a monomorphism. Thus if we assume that both $Q_{1}$ and $Q_{2}$ are in add $Q$ this shows that $\bar{f}=\bar{j} \cdot \bar{p}$ is either zero or a monomorphism, proving the lemma.

From the exact sequence $0 \rightarrow \operatorname{Ker} f \rightarrow Q_{1} \stackrel{p}{\longrightarrow} \operatorname{Im} f \rightarrow 0$ we get an exact sequence $\operatorname{Ker} f / \mathfrak{A} \operatorname{Ker} f \rightarrow Q_{1} / \mathfrak{A} Q_{1} \stackrel{\bar{p}}{\longrightarrow} \operatorname{Im} f / \mathfrak{A} \operatorname{Im} f \rightarrow 0$. Since $\operatorname{Im} f$ is a submodule of the projective module $Q_{2}$ it follows from the above lemma that $L L\left(P_{0}(\operatorname{Ker} f)\right)=L L\left(P_{1}(\operatorname{Im} f)\right)<L L\left(P_{0}(\operatorname{Im} f)\right)=L L\left(Q_{1}\right)$. If we assume that $Q_{1}$ is in add $Q$ then $L L\left(Q_{1}\right)=r$. So $L L\left(P_{0}(\operatorname{Ker} f)\right)<r$. That is, $P_{0}(\operatorname{Ker} f)$ is in add $P$. Hence $\mathfrak{A} \operatorname{Ker} f=\operatorname{Ker} f$. This proves that $\bar{p}$ is an isomorphism.

To prove that $\bar{j}$ is either zero or a monomorphism we consider the following exact sequence:

$$
\begin{aligned}
0 & \rightarrow\left(\operatorname{Im} f \cap \mathfrak{A} Q_{2}\right) / \mathfrak{A} \operatorname{Im} f \rightarrow \operatorname{Im} f / \mathfrak{A} \operatorname{Im} f \\
& \rightarrow\left(\operatorname{Im} f+\mathfrak{A} Q_{2}\right) / \mathfrak{A} Q_{2} \rightarrow 0 .
\end{aligned}
$$

The last module is precisely the image of $\bar{j}: \operatorname{Im} f / \mathfrak{A} \operatorname{Im} f \rightarrow Q_{2} / \mathfrak{A} Q_{2}$. It follows that $\bar{j}=0$ if $\left(\operatorname{Im} f+\mathfrak{A} Q_{2}\right) \subset \mathfrak{A}\left(\operatorname{Im} f+\mathfrak{A} Q_{2}\right)$, which is the same as $P_{0}\left(\operatorname{Im} f+\mathfrak{A} Q_{2}\right)$ is in add $P$. Similarly, $\bar{j}$ is a monomorphism if $\operatorname{Im} f \cap \mathfrak{A} Q_{2}=$ $\mathfrak{A}\left(\operatorname{Im} f \cap \mathfrak{A} Q_{2}\right)$ which is the same as $P_{0}\left(\operatorname{Im} f \cap \mathfrak{A} Q_{2}\right)$ is in add $P$.

We now show that either $P_{0}\left(\operatorname{Im} f+\mathfrak{A} Q_{2}\right)$ is in add $P$ or $P_{0}\left(\operatorname{Im} f \cap \mathfrak{A} Q_{2}\right)$ is in add $P$. This is a consequence of the following result applied to $X=\tau_{P} Q_{2}$, $Y=\operatorname{Im} f$.

Claim. Let $Q_{2}$ be in add $Q$ and let $X$ and $Y$ be submodules of $Q_{2}$. Suppose $P_{0}(X)$ is in add $P$ and $P_{0}(Y)$ is indecomposable and in add $Q$. Then either $P_{0}(X+Y)$ is in add $P$ or $P_{0}(X \cap Y)$ is in add $P$.

To prove this we assume that $P_{0}(X+Y)$ is not in add $P$, and consider the diagram:

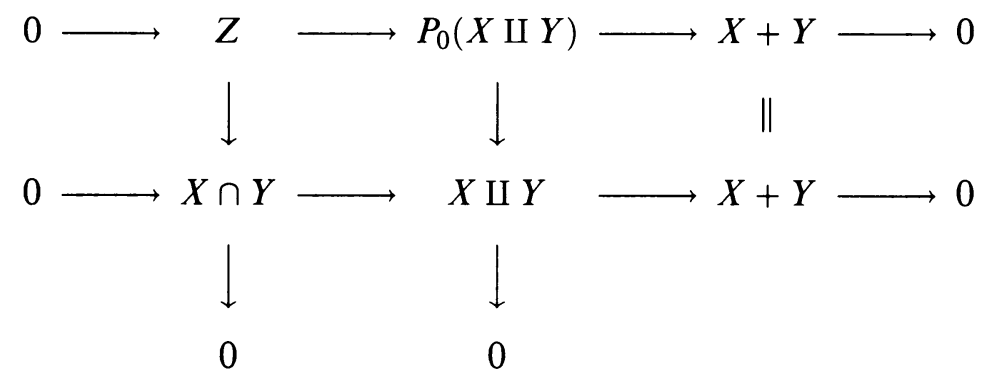

To prove that $P_{0}(X \cap Y)$ is in add $P$ it is enough to show that $P_{0}(Z)$ is in add $P$. Consider now the diagram 


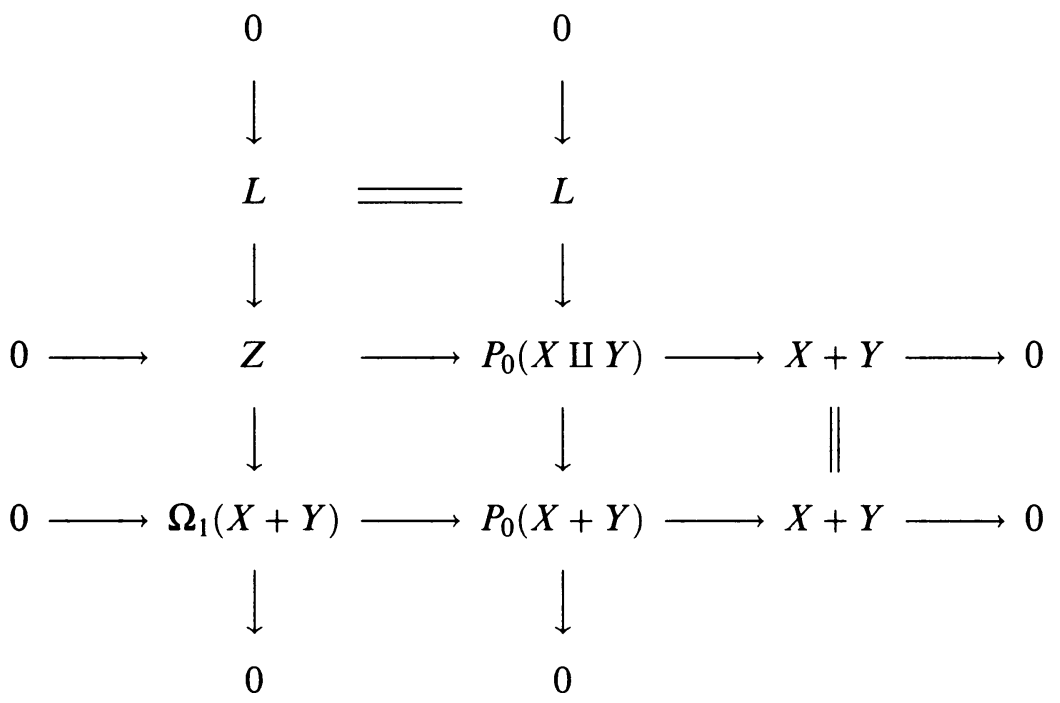

The second vertical column splits. Since $P_{0}(X+Y)$ is not in add $P$ and $P_{0}(X \amalg Y)$ has only one indecomposable direct summand not in add $P$, it follows that $L$ is in add $P$. On the other hand, we know from the preceding lemma that

$$
L L\left(P_{0}\left(\Omega_{1}(X+Y)\right)\right)=L L\left(P_{1}(X+Y)\right)<L L\left(P_{0}(X+Y)\right)
$$

since $X+Y$ is a submodule of the projective module $Q_{2}$. But $L L\left(P_{0}(X+Y)\right) \leq$ $L L\left(P_{0}(X \amalg Y)\right) \leq r$ since $P_{0}(X)$ and $P_{0}(Y)$ are in add $Q$. So

$$
L L\left(P_{0}\left(\Omega_{1}(X+Y)\right)\right)<r .
$$

That is, $P_{0}\left(\Omega_{1}(X+Y)\right)$ is in add $P$. So $P_{0}(Z)$ is in add $P$. This ends the proof of the claim and of the lemma.

\section{REFERENCES}

[A] M. Auslander, Representation theory of artin algebras. I, Comm. Algebra 1 (1974), 177-268.

[BF] W. D. Burguess and K. R. Fuller, On quasihereditary rings, 1988 (preprint).

[CE] H. Cartan and S. Eilenberg, Homological algebra, Princeton Univ. Press, Princeton, N.J., 1956.

[CPS] E. Cline, B. Parshall and L. Scott, Finite dimensional algebras and highest weight categories, J. Reine Angew. Math. 391 (1988), 277-291.

[DR1] V. Dlab and C. M. Ringel, Quasihereditary algebras, Illinois J. Math. (to appear).

[DR2] __ Every semiprimary ring is the endomorphism ring of a projective module over a quasihereditary ring, 1987 (preprint).

[PS] B. Parshall and L. Scott (Eds.), Derived categories, quasihereditary algebras and algebraic groups, Proc. Ottawa-Mosonee Workshop in Algebra, Math. Lecture Notes Series No. 3, Center for Research in Algebra and Related Topics. 9110

Department of Mathematics, Brandeis University, Waltham, Massachusetts 02254-

Departamento de Matemática, Universidad Nacional del Sur, 8000 Bahia Blanca, ARgENTINA

Department of Mathematics, Northeastern University, Boston, Massachusetts 02115 